\title{
Factors Governing the Kinetic Competition of Nitrogen and Sulfur Ligands in Cisplatin Binding to Biological Targets
}

\author{
Dirk V. Deubel* \\ Department of Chemistry and Applied Biosciences, ETH Zürich, Computational Science \\ Laboratory, USI Campus, 6900 Lugano, Switzerland \\ metals-in-medicine@phys.chem.ethz.ch
}

Supporting Information, ja0499602

\section{Tables}

Table S-1. Energy decomposition analysis at several points on the reaction coordinate of the reaction of cis- $\left[\mathrm{Pt}\left(\mathrm{NH}_{3}\right)_{2}\left(\mathrm{H}_{2} \mathrm{O}\right) \mathrm{Cl}\right]^{+}(\mathbf{1})$ with $\mathrm{L}=\mathrm{NH}_{3}$ and $\mathrm{H}_{2} \mathrm{~S}$. $\mathrm{Pt}-\mathrm{OH}_{2}$ is the distance (in $\AA$ ) between $\mathrm{Pt}$ and the $\mathrm{O}$ of the aqua ligand that is replaced by the nucleophile. The energy contributions (in $\mathrm{kcal} / \mathrm{mol}$ ) are strain energy (Str, separately listed for $\mathbf{1}$ and $\mathrm{L}$ ), stabilizing orbital interactions (Orb), electrostatic interactions (Elst), Pauli repulsion (Pauli), interaction energy $(\mathrm{Int}=$ Pauli + Elst + Orb $)$, and the energy sum $(\mathrm{Sum}=\mathrm{Str}+$ Int). The activation energy is the energy sum at the transition state. These results are displayed in Figure 3.

\begin{tabular}{|c|c|c|c|c|c|c|}
\hline \multirow[t]{2}{*}{$\mathrm{Pt}-\mathrm{OH}_{2}$} & \multicolumn{2}{|c|}{2.36} & \multicolumn{2}{|c|}{2.42} & \multicolumn{2}{|c|}{2.48} \\
\hline & $\mathrm{NH}_{3}$ & $\mathrm{H}_{2} \mathrm{~S}$ & $\mathrm{NH}_{3}$ & $\mathrm{H}_{2} \mathrm{~S}$ & $\mathrm{NH}_{3}$ & $\mathrm{H}_{2} \mathrm{~S}$ \\
\hline Str 1 & 0.8 & 0.4 & 0.7 & 0.4 & 0.9 & 0.4 \\
\hline Str L & 15.6 & 15.6 & 17.8 & 18.3 & 20.7 & 21.2 \\
\hline Str total & 16.5 & 16.1 & 18.4 & 18.7 & 21.6 & 21.6 \\
\hline Elst & 29.0 & 26.3 & 34.6 & 30.4 & 41.7 & 35.2 \\
\hline Pauli & -26.9 & -19.8 & -31.3 & -23.2 & -37.3 & -27.2 \\
\hline Orb & -13.4 & -12.6 & -16.5 & -15.7 & -20.7 & -19.5 \\
\hline Int & -11.3 & -6.1 & -13.3 & -8.5 & -16.3 & -11.5 \\
\hline Sum & 5.2 & 10.0 & 5.2 & 10.2 & 5.4 & 10.1 \\
\hline
\end{tabular}


Table S-2. Energy decomposition analysis at several points on the reaction coordinate of the reaction of cis- $\left[\mathrm{Pt}\left(\mathrm{NH}_{3}\right)_{2}\left(\mathrm{H}_{2} \mathrm{O}\right)_{2}\right]^{+}(2)$ with $\mathrm{L}=\mathrm{NH}_{3}$ and $\mathrm{H}_{2} \mathrm{~S}$. $\mathrm{Pt}_{-}-\mathrm{OH}_{2}$ is the distance (in $\AA$ ) between $\mathrm{Pt}$ and the $\mathrm{O}$ of the aqua ligand that is replaced by the nucleophile. The energy contributions (in $\mathrm{kcal} / \mathrm{mol}$ ) are strain energy (Str, separately listed for $\mathbf{2}$ and $\mathrm{L}$ ), stabilizing orbital interactions (Orb), electrostatic interactions (Elst), Pauli repulsion (Pauli), interaction energy $(\mathrm{Int}=$ Pauli + Elst + Orb $)$, and the energy sum $(\mathrm{Sum}=\mathrm{Str}+$ Int). The activation energy is the energy sum at the transition state. These results are displayed in Figure 4.

\begin{tabular}{|c|c|c|c|c|c|c|}
\hline \multirow[t]{2}{*}{$\mathrm{Pt}-\mathrm{OH}_{2}$} & \multicolumn{2}{|c|}{2.36} & \multicolumn{2}{|c|}{2.42} & \multicolumn{2}{|c|}{2.48} \\
\hline & $\mathrm{NH}_{3}$ & $\mathrm{H}_{2} \mathrm{~S}$ & $\mathrm{NH}_{3}$ & $\mathrm{H}_{2} \mathrm{~S}$ & $\mathrm{NH}_{3}$ & $\mathrm{H}_{2} \mathrm{~S}$ \\
\hline Str 2 & 0.7 & 0.7 & 0.7 & 0.5 & 0.7 & 0.5 \\
\hline Str L & 14.4 & 15.6 & 17.4 & 18.2 & 20.9 & 21.0 \\
\hline Str total & 15.1 & 16.3 & 18.0 & 18.7 & 21.6 & 21.5 \\
\hline Elst & 23.6 & 20.1 & 28.6 & 23.1 & 35.1 & 26.5 \\
\hline Pauli & -27.8 & -17.2 & -32.1 & -19.8 & -38.1 & -22.7 \\
\hline Orb & -15.8 & -16.9 & -19.3 & -19.9 & -23.9 & -23.6 \\
\hline Int & -20.0 & -14.0 & -22.9 & -16.6 & -26.9 & -19.8 \\
\hline Sum & -4.9 & 2.3 & -4.9 & 2.1 & -5.3 & 1.7 \\
\hline
\end{tabular}

Table S-3. Calculated reaction free energies $\left(\Delta \mathrm{G}_{\mathrm{r}}\right)$ for the nucleophilic substitution of the aqua ligand in $c i s-\left[\mathrm{Pt}\left(\mathrm{NH}_{3}\right)_{2}\left(\mathrm{H}_{2} \mathrm{O}\right) \mathrm{Cl}\right]^{+}(\mathbf{1})$ and $c i s-\left[\mathrm{Pt}\left(\mathrm{NH}_{3}\right)_{2}\left(\mathrm{H}_{2} \mathrm{O}\right)_{2}\right]^{2+}(\mathbf{2})$ by nitrogen and sulfur ligands, $\mathrm{mG}=9$-methylguanine, $\mathrm{H}=5$-methylimidazole, $\mathrm{M}=$ dimethylsulfide, $\mathrm{C}^{-}$ $=$ methanethiolate. These results are displayed in Figure 6 .

\begin{tabular}{|c|c|c|c|c|c|c|c|c|}
\hline \multirow[b]{2}{*}{$\varepsilon$} & \multicolumn{4}{|c|}{1} & \multicolumn{4}{|c|}{2} \\
\hline & $\mathrm{mG}$ & $\mathrm{H}$ & $\mathrm{M}$ & $\mathrm{C}^{-}$ & $\mathrm{mG}^{\mathrm{a}}$ & $\mathrm{H}$ & $\mathrm{M}$ & $\mathrm{C}^{-}$ \\
\hline 1 & -39.1 & -30.4 & -21.0 & -138.8 & -69.8 & -50.5 & -29.5 & -239.1 \\
\hline 3 & -23.0 & -20.8 & -15.1 & -56.1 & -32.0 & -27.5 & -17.1 & -89.3 \\
\hline 9 & -17.0 & -17.9 & -13.6 & -28.8 & -19.8 & -20.6 & -14.2 & -40.7 \\
\hline 24 & -14.8 & -16.9 & -13.0 & -20.4 & -15.8 & -18.2 & -13.5 & -25.6 \\
\hline 80 & -14.0 & -16.6 & -12.9 & -17.0 & -14.3 & -17.4 & -13.5 & -19.4 \\
\hline
\end{tabular}

${ }^{a}$ This structure contains a $\left(\mathrm{Pt}\right.$-aqua) $\mathrm{O}-\mathrm{H} \ldots \mathrm{O}^{6}(\mathrm{mG})$ hydrogen bond. The isomer involving a $(\mathrm{Pt}$-ammine $) \mathrm{N}-\mathrm{H} . . . \mathrm{O}^{6}(\mathrm{mG})$ hydrogen bond is between $5.3 \mathrm{kcal} / \mathrm{mol}(\varepsilon=1)$ and 1.9 $\mathrm{kcal} / \mathrm{mol}(\varepsilon=80)$ higher in energy. 
Table S-4. Calculated activation free energies $\left(\Delta \mathrm{G}_{\mathrm{a}}\right)$ for the nucleophilic substitution of the aqua ligand in $c i s-\left[\mathrm{Pt}\left(\mathrm{NH}_{3}\right)_{2}\left(\mathrm{H}_{2} \mathrm{O}\right) \mathrm{Cl}\right]^{+}(\mathbf{1})$ and $c i s-\left[\mathrm{Pt}\left(\mathrm{NH}_{3}\right)_{2}\left(\mathrm{H}_{2} \mathrm{O}\right)_{2}\right]^{2+}(\mathbf{2})$ by nitrogen and sulfur ligands, $\mathrm{mG}=9$-methylguanine, $\mathrm{H}=5$-methylimidazole, $\mathrm{M}=$ dimethylsulfide, $\mathrm{C}^{-}=$methanethiolate. These results are displayed in Figure 7.

\begin{tabular}{|c|c|c|c|c|c|c|c|c|}
\hline \multirow[b]{2}{*}{$\varepsilon$} & \multicolumn{4}{|c|}{1} & \multicolumn{4}{|c|}{2} \\
\hline & $\mathrm{mG}$ & $\mathrm{H}$ & $\mathrm{M}$ & $\mathrm{C}^{-}$ & $\mathrm{mG}^{\mathrm{a}}$ & $\mathrm{H}$ & $\mathrm{M}$ & $\mathrm{C}^{-}$ \\
\hline 1 & -8.2 & 3.6 & 12.7 & -94.4 & -44.5 & -14.8 & 0.6 & -191.2 \\
\hline 3 & 12.2 & 18.3 & 21.9 & -10.1 & 1.3 & 13.9 & 20.4 & -40.8 \\
\hline 9 & 20.8 & 24.0 & 25.4 & 18.0 & 17.1 & 23.5 & 26.2 & 7.9 \\
\hline 24 & 24.0 & 26.2 & 26.6 & 26.9 & 22.3 & 26.8 & 28.0 & 22.8 \\
\hline 80 & 25.2 & 26.9 & 27.1 & 30.4 & 24.4 & 28.0 & 28.7 & 28.9 \\
\hline
\end{tabular}

${ }^{\mathrm{a}}$ This structure contains a $\left(\mathrm{Pt}\right.$-aqua) $\mathrm{O}-\mathrm{H} \ldots \mathrm{O}^{6}(\mathrm{mG})$ hydrogen bond. The isomer involving a $(\mathrm{Pt}$-ammine $) \mathrm{N}-\mathrm{H} \ldots \mathrm{O}^{6}(\mathrm{mG})$ hydrogen bond is between $9.8 \mathrm{kcal} / \mathrm{mol}(\varepsilon=1)$ and 4.2 $\mathrm{kcal} / \mathrm{mol}(\varepsilon=80)$ higher in energy.

Table S-5. Dependence of the absolute and relative $\mathrm{pK}_{\mathrm{a}}(\mathrm{raw})$ of methanethiol $(\mathrm{MeSH})$ and benzenethiol $(\mathrm{PhSH})$ on the atomic radius $\mathrm{r}(\mathrm{S})$ (in $\AA$ ) at sulfur in the PoissonBoltzmann calculations. The $\mathrm{pK}_{\mathrm{a}}$ values were calculated according to: Klicic, J. J.; Friesner, R. A.; Liu, S.-Y.; Guida, W. C. J. Phys. Chem. A. 2002, 106, 1327. $\Delta \mathrm{G}^{1}$ is 350.1 $\mathrm{kcal} / \mathrm{mol}(\mathrm{MeSH})$ or $333.2 \mathrm{kcal} / \mathrm{mol}(\mathrm{PhSH})$ at B3LYP/III+//II-. $\mathrm{G}_{\text {solv }}{ }^{80}\left(\mathrm{H}^{+}\right)$is -260.9 $\mathrm{kcal} / \mathrm{mol}$ as recommended in: Chambers, C. C.; Hawkins, G. D.; Cramer, C. J.; Truhlar, D. G. J. Phys. Chem. 1996, 100, 16385. The experimental $\mathrm{pK}_{\mathrm{a}}$ values are $10.3(\mathrm{MeSH})$ and 6.6 $(\mathrm{PhSH})$, see Klicic et al. $\Sigma\left(\Delta \mathrm{pK}_{\mathrm{a}}\right)$ is the error sum, $\Sigma\left(\Delta \mathrm{pK}_{\mathrm{a}}\right)=\mid \mathrm{pK}_{\mathrm{a}}(\mathrm{calc}, \mathrm{MeSH})-$ $\mathrm{pK}_{\mathrm{a}}(\exp , \mathrm{MeSH})|+| \mathrm{pK}_{\mathrm{a}}(\mathrm{calc}, \mathrm{PhSH})-\mathrm{pK}_{\mathrm{a}}(\exp , \mathrm{PhSH}) \mid . \Sigma\left(\Delta \mathrm{pK}_{\mathrm{a}}\right)$ is smallest at $\mathrm{r}(\mathrm{S})=$ 1.90 .

\begin{tabular}{|c|c|c|c|c|c|c|c|c|c|}
\hline \multirow[b]{2}{*}{$\mathrm{r}(\mathrm{S})$} & \multicolumn{4}{|c|}{$\mathrm{R}=\mathrm{Me}$} & \multicolumn{4}{|c|}{$\mathrm{R}=\mathrm{Ph}$} & \multirow[b]{2}{*}{$\Sigma\left(\Delta \mathrm{pK}_{\mathrm{a}}\right)$} \\
\hline & $\begin{array}{l}\mathrm{G}_{\text {solv }}^{80} \\
\text { (RSH) }\end{array}$ & $\begin{array}{l}\mathrm{G}_{\text {solv }}{ }^{80} \\
\left(\mathrm{RS}^{-}\right)\end{array}$ & $\Delta \mathrm{G}^{80}$ & $\mathrm{pK}_{\mathrm{a}}$ & $\begin{array}{l}\mathrm{G}_{\text {solv }}{ }^{0} \\
\text { (RSH) }\end{array}$ & $\begin{array}{l}\mathrm{G}_{\text {solv }}{ }^{80} \\
\left(\mathrm{RS}^{-}\right)\end{array}$ & $\Delta \mathrm{G}^{80}$ & $\mathrm{pK}_{\mathrm{a}}$ & \\
\hline 1.50 & -2.6 & -85.2 & 6.6 & 4.8 & -4.8 & -70.7 & 6.4 & 4.7 & 7.4 \\
\hline 1.60 & -3.3 & -85.4 & 7.1 & 5.2 & -5.4 & -70.9 & 6.8 & 5.0 & 6.7 \\
\hline 1.70 & -3.7 & -84.1 & 8.8 & 6.4 & -5.7 & -70.1 & 7.9 & 5.8 & 4.7 \\
\hline 1.80 & -3.7 & -81.8 & 11.1 & 8.1 & -5.7 & -68.6 & 9.5 & 7.0 & 2.5 \\
\hline 1.90 & -3.7 & -79.2 & 13.6 & 10.0 & -5.7 & -66.7 & 11.3 & 8.3 & 2.0 \\
\hline 2.00 & -3.5 & -76.4 & 16.3 & 11.9 & -5.6 & -64.8 & 13.1 & 9.6 & 4.6 \\
\hline 2.10 & -3.3 & -73.6 & 18.9 & 13.9 & -5.5 & -62.8 & 15.1 & 11.0 & 8.0 \\
\hline
\end{tabular}


Table S-6. Calculated $\mathrm{pK}_{\mathrm{a}}^{\varepsilon}(\mathrm{raw})$ of methanethiol (MeSH) in dependence of the dielectric constant $\varepsilon$.

\begin{tabular}{rrrrrr}
$\varepsilon$ & $\begin{array}{c}\mathrm{G}_{\text {solv }}{ }^{\varepsilon} \\
(\mathrm{RSH})\end{array}$ & $\begin{array}{r}\mathrm{G}_{\text {solv }}{ }^{\varepsilon} \\
(\mathrm{RS})\end{array}$ & $\begin{array}{c}\mathrm{G}_{\text {solv }}{ }^{\varepsilon} \\
\left(\mathrm{H}^{+}\right)\end{array}$ & $\Delta \mathrm{G}^{\varepsilon}$ & $\mathrm{pK}_{\mathrm{a}}{ }^{\varepsilon}$ \\
\hline 1 & -0.0 & 0.0 & 0.0 & 350.1 & 256.7 \\
3 & -2.1 & -51.6 & -176.1 & 124.4 & 91.2 \\
9 & -3.1 & -70.5 & -234.8 & 47.9 & 35.1 \\
24 & -3.5 & -76.6 & -253.2 & 23.8 & 17.4 \\
80 & -3.7 & -79.2 & -260.9 & 13.6 & 10.0 \\
\hline
\end{tabular}

\section{Calculated Structures of Transition States and Products}

Figure S-1. Calculated structures (B3LYP) of the transition states for the reactions of $\mathbf{1}$ with $\mathrm{N}$ and $\mathrm{S}$ ligands.

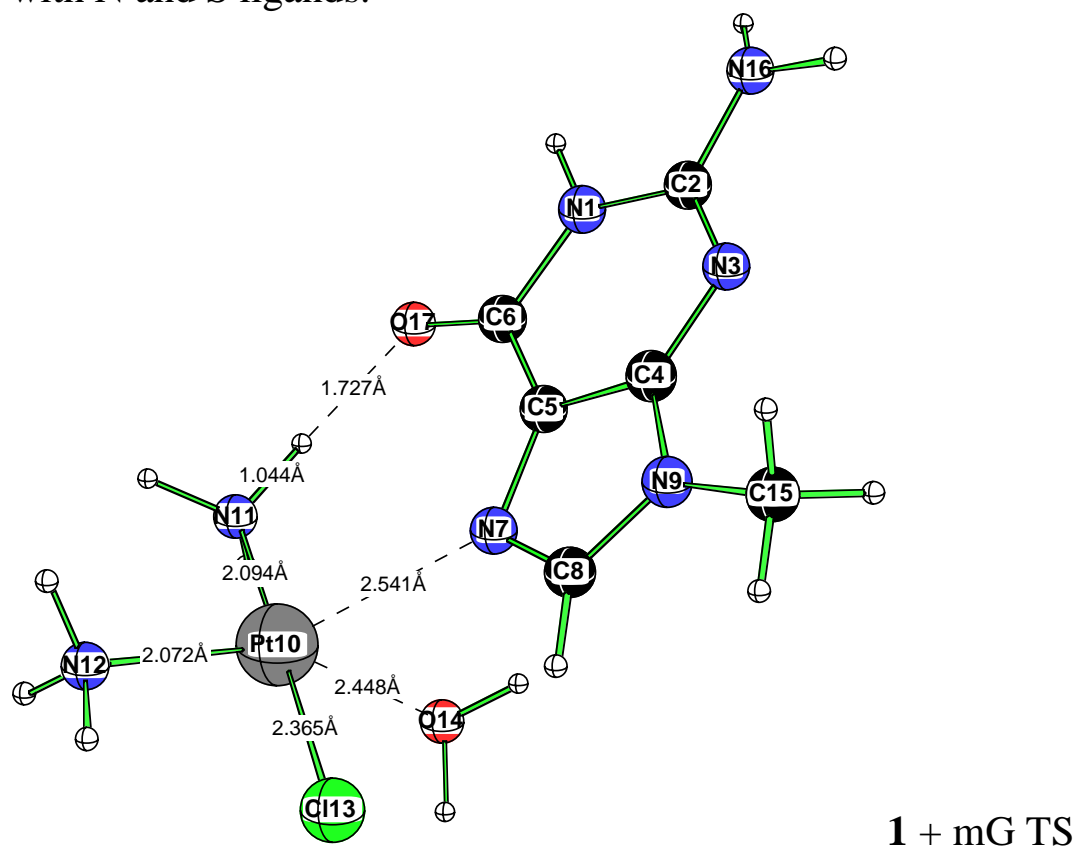




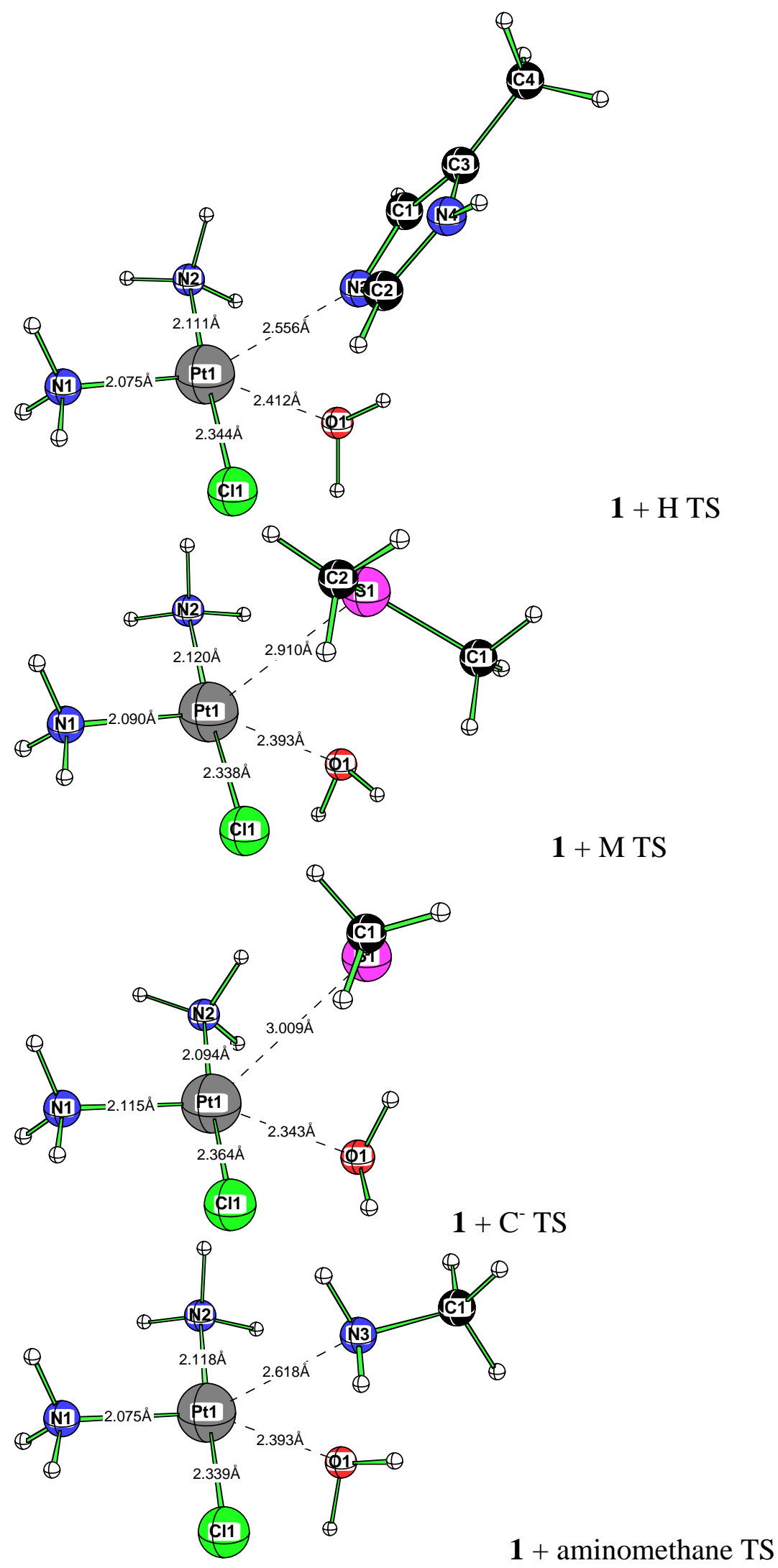




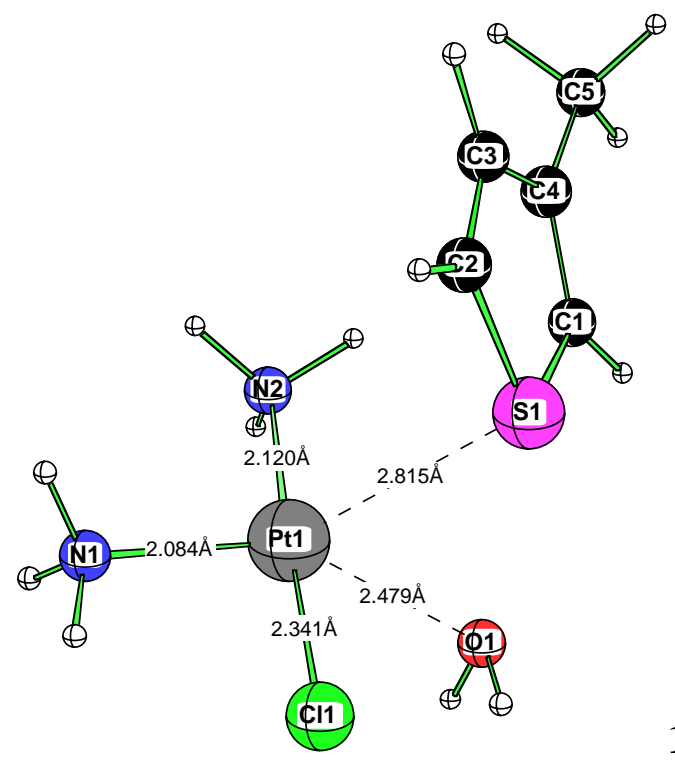

$1+3$-methylthiophene TS

Figure S-2. Calculated structures (B3LYP) of the products of the reactions of $\mathbf{1}$ with $\mathrm{N}$ and S ligands.

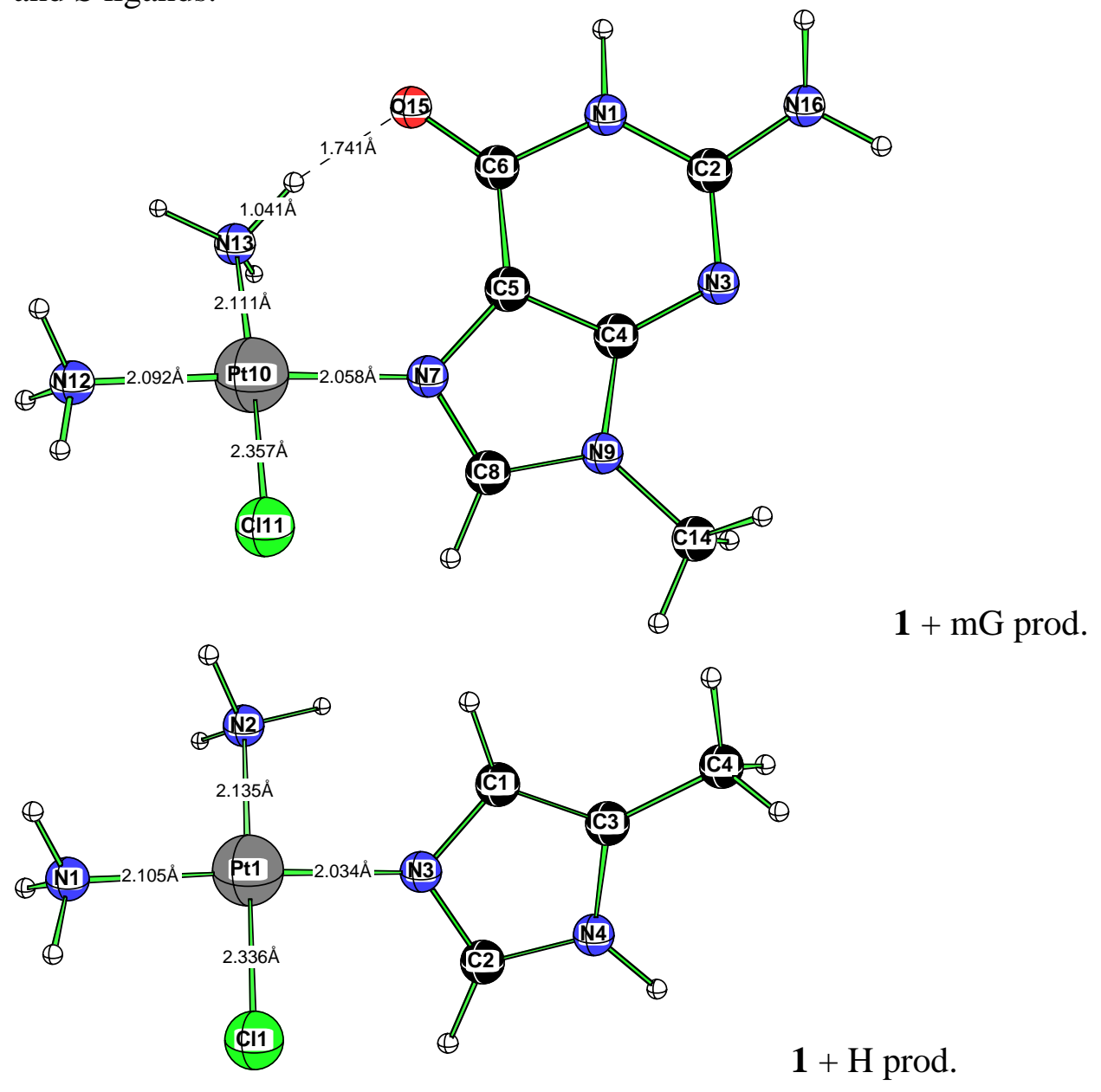



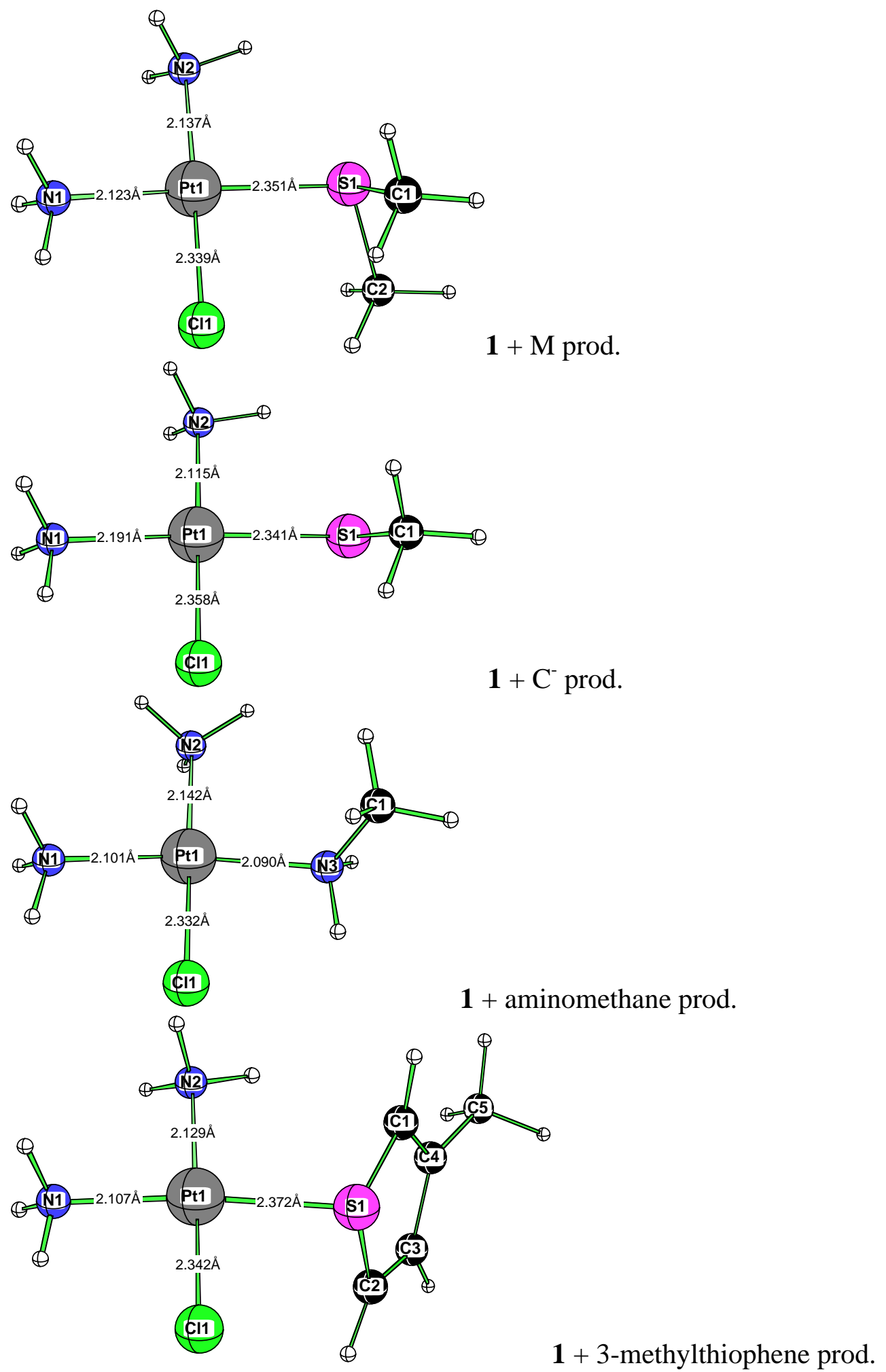

Figure S-3. Calculated structures (B3LYP) of the transition states for the reactions of 2 with $\mathrm{N}$ and $\mathrm{S}$ ligands. 


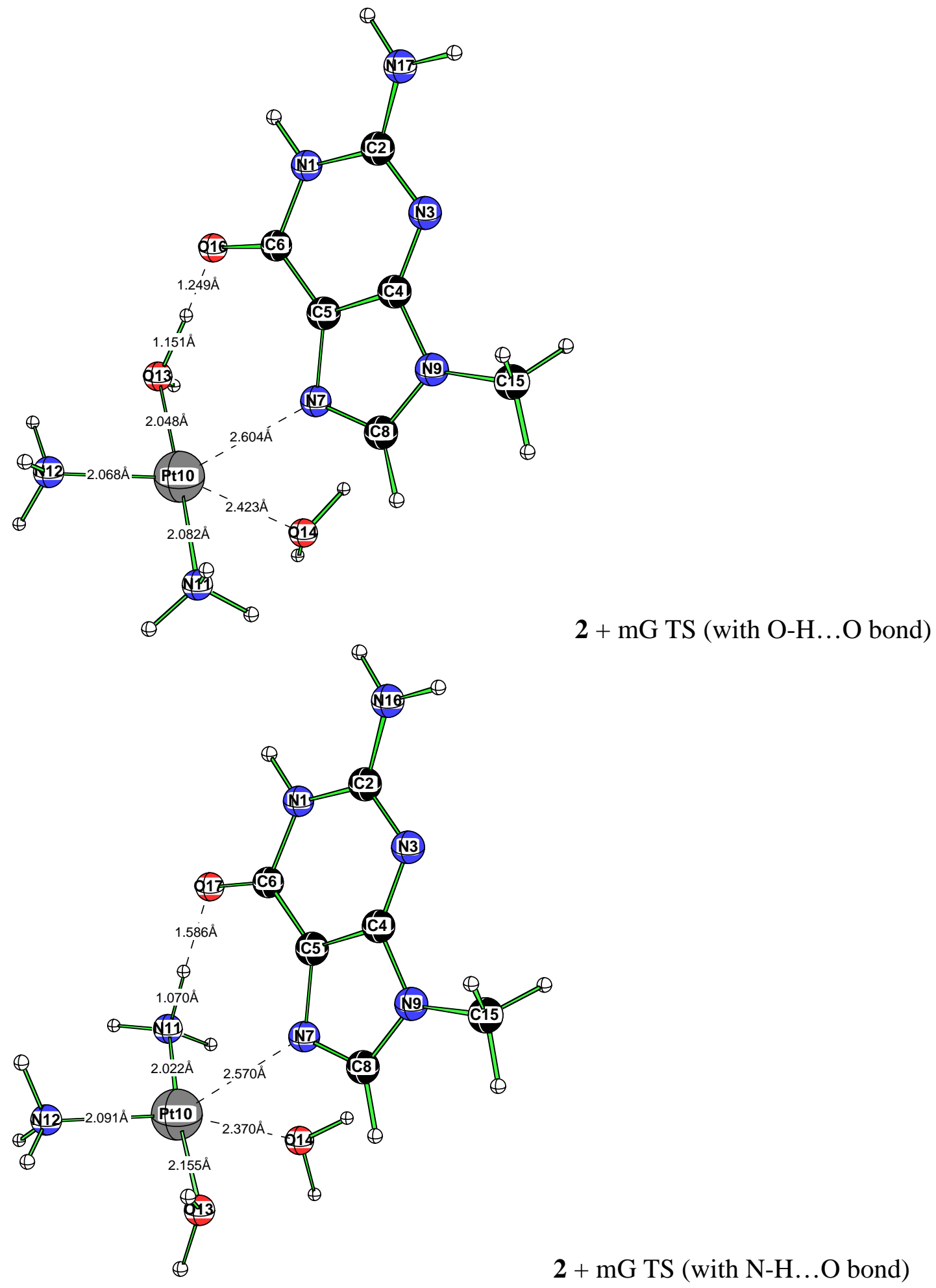




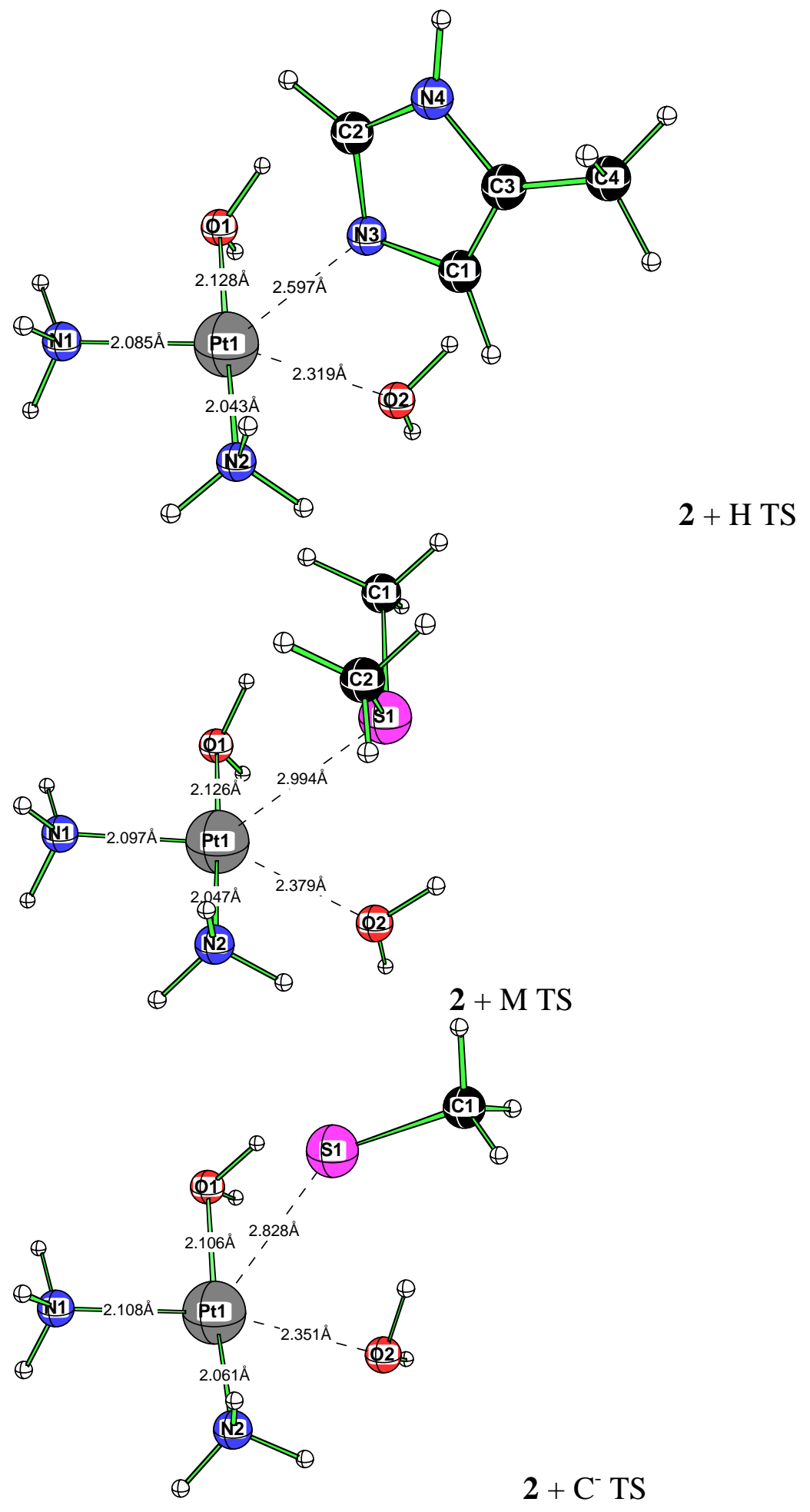


Figure S-4. Calculated structures (B3LYP) of the products of the reactions of $\mathbf{2}$ with $\mathrm{N}$ and S ligands.

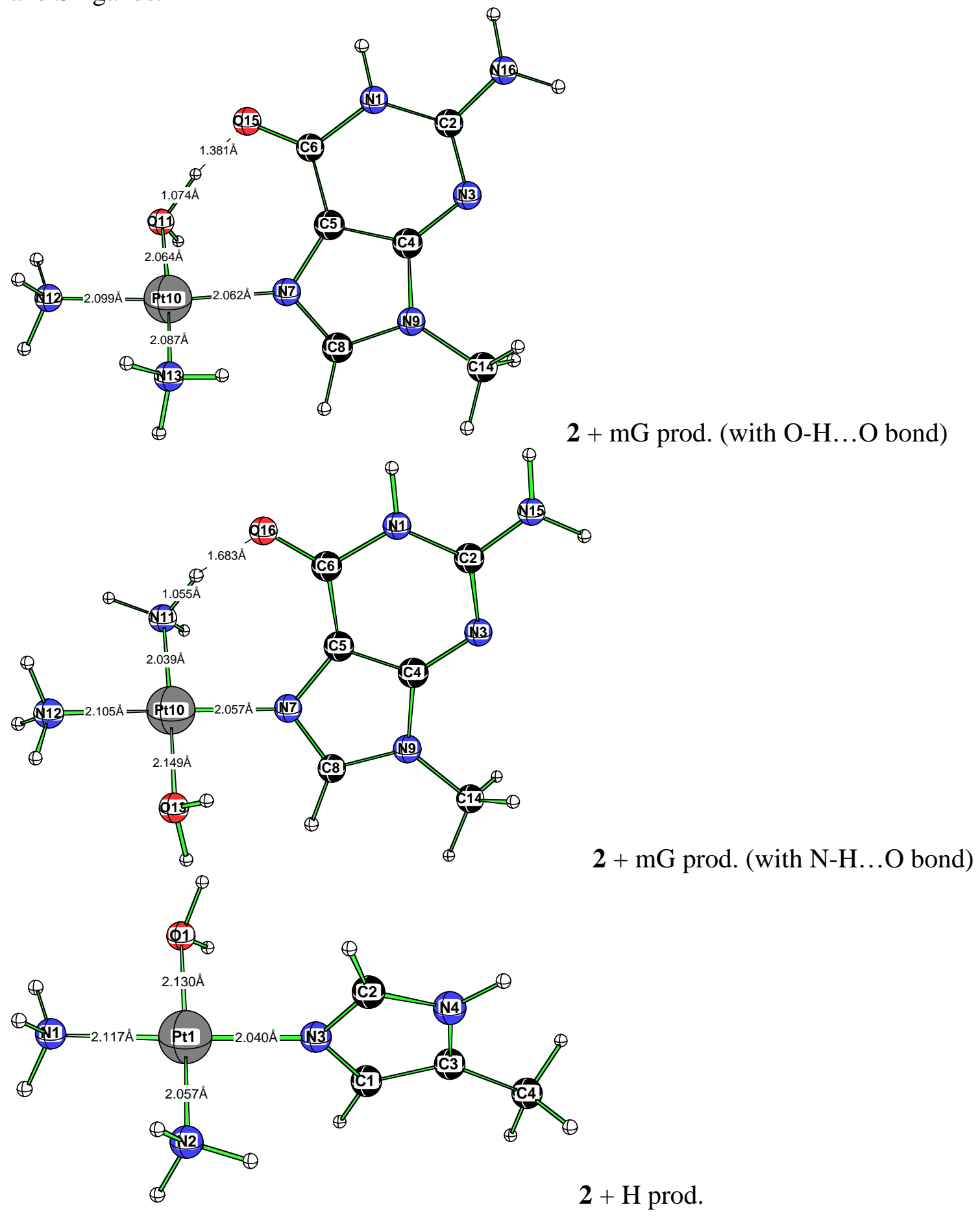




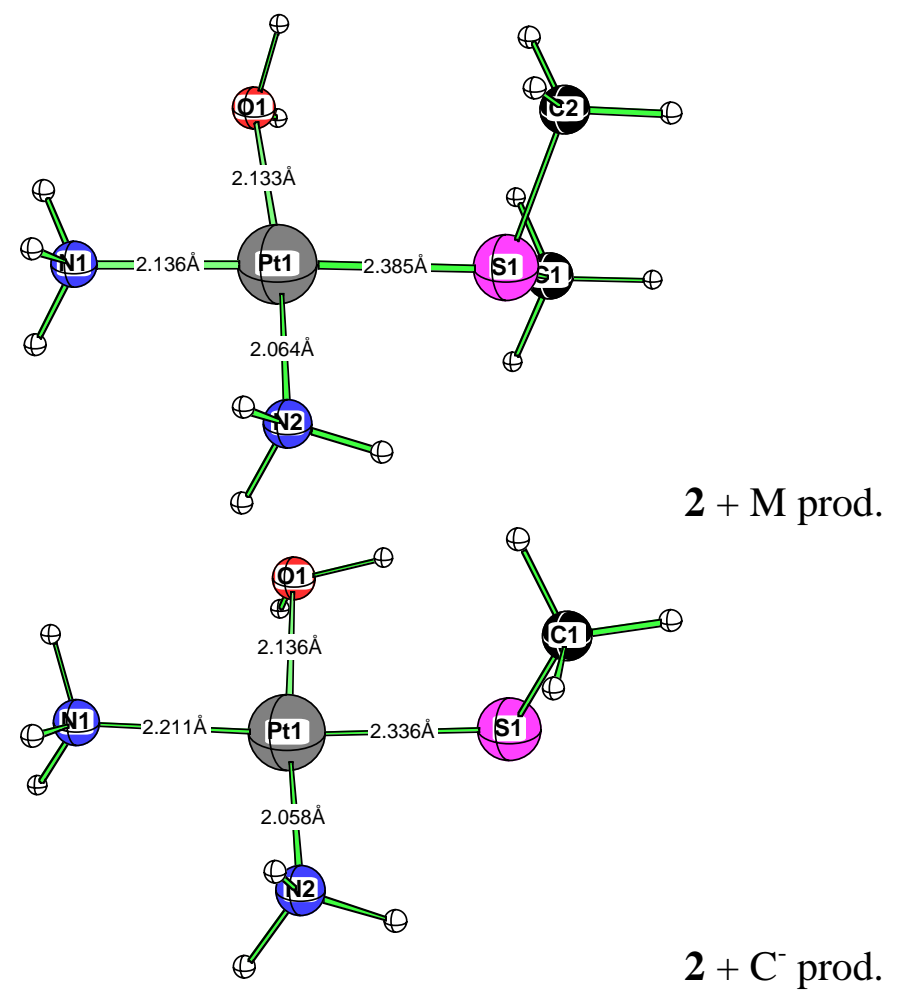

Figure S-5. Calculated structures (BLYP) of the transition states for the reactions of $\mathbf{1}$ with $\mathrm{NH}_{3}$ and $\mathrm{H}_{2} \mathrm{~S}$.
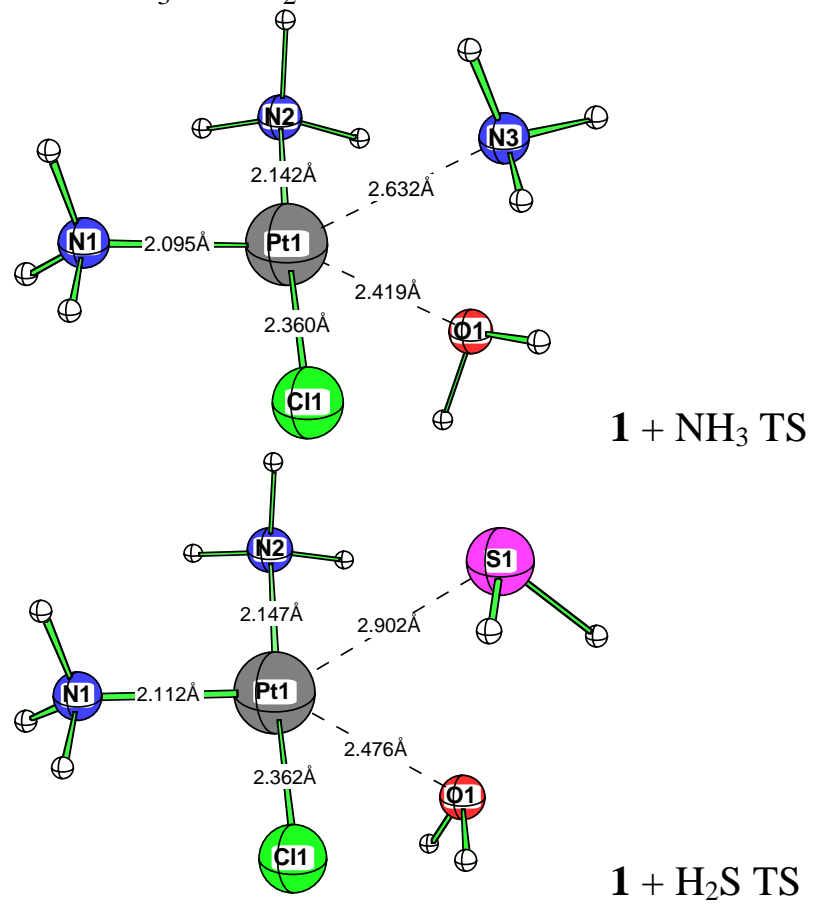
Figure S-6. Calculated structures (BLYP) of the transition states for the reactions of 2 with $\mathrm{NH}_{3}$ and $\mathrm{H}_{2} \mathrm{~S}$.

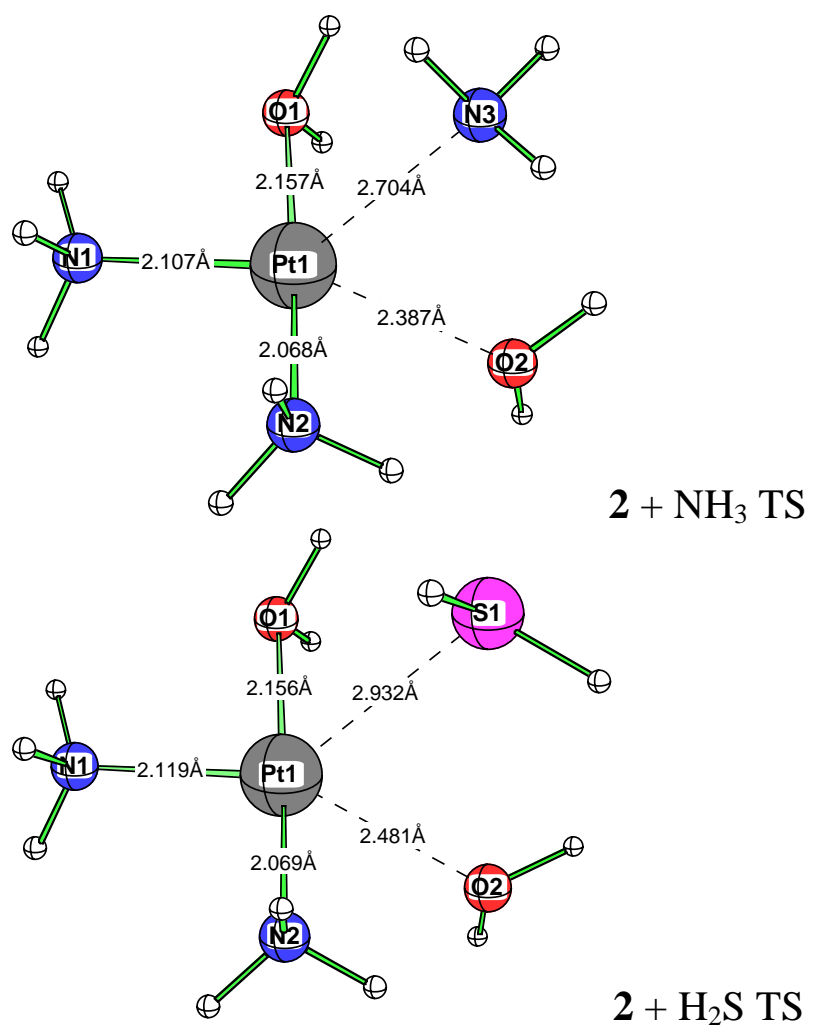




\section{Cartesian Coordinates}

$1+\mathrm{mG}$ TS

$\begin{array}{llll}\text { Pt } & -1.80844 & -0.26592 & -0.06202\end{array}$

$\begin{array}{llll}\mathrm{N} & -1.26496 & -2.28132 & 0.10060\end{array}$

$\begin{array}{llll}\mathrm{N} & -3.28774 & -0.56003 & -1.48250\end{array}$

$\begin{array}{llll}\mathrm{Cl} & -2.50218 & 1.99465 & -0.05513\end{array}$

$\begin{array}{llll}\mathrm{N} & 0.59044 & 0.51434 & 0.24640\end{array}$

$\begin{array}{llll}\mathrm{O} & -1.25414 & -0.17422 & 2.32031\end{array}$

$\begin{array}{llll}\text { C } & 0.85131 & 1.80568 & 0.14653\end{array}$

$\begin{array}{llll}\text { C } & 1.82631 & -0.10695 & 0.11739\end{array}$

$\begin{array}{llll}\mathrm{N} & 2.18340 & 2.06855 & -0.03017\end{array}$

$\begin{array}{llll}\text { C } & 2.82557 & 0.85321 & -0.05101\end{array}$

$\begin{array}{llll}\text { C } & 2.81666 & 3.37711 & -0.17979\end{array}$

$\begin{array}{llll}\mathrm{N} & 4.15176 & 0.66988 & -0.19901\end{array}$

$\begin{array}{llll}\text { C } & 4.50377 & -0.60024 & -0.20119\end{array}$

$\begin{array}{llll}\mathrm{N} & 3.59463 & -1.62946 & -0.06395\end{array}$

$\begin{array}{llll}\text { C } & 2.19834 & -1.48480 & 0.08983\end{array}$

$\begin{array}{llll}\mathrm{N} & 5.80435 & -0.93786 & -0.38875\end{array}$

$\begin{array}{llll}\mathrm{O} & 1.49631 & -2.50091 & 0.16514\end{array}$

$\mathrm{H} \quad-0.22481 \quad-2.36990 \quad 0.10292$

$\begin{array}{llll}\mathrm{H} & -1.63013 & -2.90921 & -0.61317\end{array}$

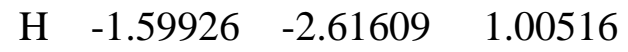

$\begin{array}{llll}\mathrm{H} & 3.90259 & -2.59455 & -0.12862\end{array}$

$\mathrm{H} \quad 6.15548 \quad-1.84458 \quad-0.11639$

$\begin{array}{llll}\mathrm{H} & 6.46567 & -0.17316 & -0.37735\end{array}$

$\begin{array}{llll}\mathrm{H} & 0.10097 & 2.58387 & 0.17407\end{array}$

$\begin{array}{llll}\mathrm{H} & -1.89564 & 0.49152 & 2.62570\end{array}$

$\begin{array}{llll}\mathrm{H} & -0.40440 & 0.29641 & 2.25258\end{array}$

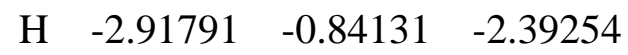

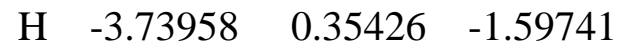

$\begin{array}{llll}\mathrm{H} & -4.00584 & -1.22949 & -1.20016\end{array}$

$\begin{array}{llll}\mathrm{H} & 3.53470 & 3.53838 & 0.62770\end{array}$

$\begin{array}{llll}\mathrm{H} & 3.33767 & 3.42943 & -1.13847\end{array}$

$\begin{array}{llll}\mathrm{H} & 2.04336 & 4.14562 & -0.14159\end{array}$

$1+\mathrm{H}$ TS

$\begin{array}{rrrr}\mathrm{Pt} & 1.02147 & -0.21022 & 0.04851 \\ \mathrm{~N} & 2.55264 & -0.05234 & 1.43947 \\ \mathrm{~N} & 0.67803 & -2.28443 & 0.23976 \\ \mathrm{Cl} & 1.37336 & 2.07681 & -0.32724 \\ \mathrm{~N} & -1.49854 & 0.12884 & -0.20848 \\ \mathrm{O} & 0.25908 & -0.59735 & -2.20730 \\ \mathrm{C} & -2.59319 & -0.72446 & -0.21541 \\ \mathrm{C} & -1.95452 & 1.30106 & 0.20918 \\ \mathrm{H} & -2.51629 & -1.75233 & -0.54257\end{array}$

$\begin{array}{rrrr}\mathrm{H} & -1.35853 & 2.19333 & 0.33375 \\ \mathrm{~N} & -3.28315 & 1.22723 & 0.46176 \\ \mathrm{C} & -3.72136 & -0.06046 & 0.19658 \\ \mathrm{H} & -0.15177 & -2.47040 & 0.80661 \\ \mathrm{H} & 1.44306 & -2.84483 & 0.61768 \\ \mathrm{H} & 0.49277 & -2.62224 & -0.70891 \\ \mathrm{C} & -5.14286 & -0.49007 & 0.35453 \\ \mathrm{H} & -3.86148 & 1.99213 & 0.78507 \\ \mathrm{H} & 2.83730 & 0.93391 & 1.41326 \\ \mathrm{H} & 3.37462 & -0.61567 & 1.21364 \\ \mathrm{H} & 2.26208 & -0.26750 & 2.39524 \\ \mathrm{H} & -0.57215 & -0.09660 & -2.09427 \\ \mathrm{H} & 0.83599 & -0.04063 & -2.75985 \\ \mathrm{H} & -5.24633 & -1.54473 & 0.08745 \\ \mathrm{H} & -5.81430 & 0.08522 & -0.29404 \\ \mathrm{H} & -5.49084 & -0.37066 & 1.38762\end{array}$

$1+\mathrm{M}$ TS

$\begin{array}{llll}\text { Pt } & 0.66556 & -0.13161 & -0.02640\end{array}$

$\begin{array}{llll}\mathrm{N} & 2.26007 & 0.54137 & 1.14604\end{array}$

$\begin{array}{llll}\mathrm{N} & 0.91030 & -2.20541 & 0.33831\end{array}$

$\begin{array}{llll}\mathrm{Cl} & 0.26931 & 2.11488 & -0.53866\end{array}$

$\begin{array}{llll}\text { S } & -2.14641 & -0.61387 & 0.54591\end{array}$

$\begin{array}{llll}\mathrm{O} & -0.34008 & -0.68554 & -2.12543\end{array}$

$\begin{array}{llll}\mathrm{H} & 0.45114 & -2.49222 & 1.20550\end{array}$

$\begin{array}{llll}\mathrm{H} & 1.87377 & -2.54312 & 0.37052\end{array}$

$\begin{array}{llll}\mathrm{H} & 0.44078 & -2.70740 & -0.41991\end{array}$

$\begin{array}{llll}\mathrm{H} & 2.25824 & 1.56237 & 1.04290\end{array}$

$\begin{array}{llll}\mathrm{H} & 3.17165 & 0.20240 & 0.83335\end{array}$

$\begin{array}{llll}\mathrm{H} & 2.15877 & 0.32916 & 2.14019\end{array}$

$\begin{array}{llll}\mathrm{H} & -0.86505 & 0.12576 & -2.26007\end{array}$

$\begin{array}{llll}\mathrm{H} & 0.38500 & -0.60547 & -2.77126\end{array}$

$\begin{array}{llll}\text { C } & -3.23328 & 0.00040 & -0.79017\end{array}$

$\begin{array}{llll}\text { C } & -2.40024 & 0.70306 & 1.78829\end{array}$

$\begin{array}{llll}\mathrm{H} & -4.26524 & 0.04216 & -0.43199\end{array}$

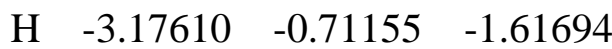

$\begin{array}{llll}\mathrm{H} & -2.91947 & 0.99609 & -1.11829\end{array}$

$\begin{array}{llll}\mathrm{H} & -3.46213 & 0.76821 & 2.03922\end{array}$

$\begin{array}{llll}\mathrm{H} & -2.03170 & 1.65931 & 1.41009\end{array}$

$\begin{array}{llll}\mathrm{H} & -1.84022 & 0.42026 & 2.68282\end{array}$

$\mathbf{1}+\mathrm{C}^{-} \mathrm{TS}$

$\begin{array}{llll}\text { Pt } & 0.45946 & -0.23107 & -0.05173\end{array}$

$\begin{array}{llll}\mathrm{N} & 2.05559 & -0.36483 & 1.32975\end{array}$

$\begin{array}{llll}\mathrm{N} & -0.17129 & -2.22640 & 0.03271\end{array}$

$\begin{array}{llll}\text { Cl } & 1.02049 & 2.06187 & -0.18253\end{array}$ 


$\begin{array}{rrrc}\mathrm{S} & -2.54461 & -0.23176 & 0.12794 \\ \mathrm{O} & -0.60577 & 0.04469 & -2.11999 \\ \mathrm{H} & -1.12794 & -2.11621 & 0.41083 \\ \mathrm{H} & 0.36262 & -2.87915 & 0.60630 \\ \mathrm{H} & -0.23997 & -2.60751 & -0.91257 \\ \mathrm{H} & 2.44496 & 0.58337 & 1.34303 \\ \mathrm{H} & 2.79575 & -1.01173 & 1.05677 \\ \mathrm{H} & 1.74573 & -0.59319 & 2.27459 \\ \mathrm{H} & -1.48792 & 0.17548 & -1.64933 \\ \mathrm{H} & -0.29550 & 0.94494 & -2.33247 \\ \mathrm{C} & -2.76219 & 1.22961 & 1.22682 \\ \mathrm{H} & -3.71810 & 1.71378 & 1.00409 \\ \mathrm{H} & -1.95649 & 1.95243 & 1.07489 \\ \mathrm{H} & -2.76620 & 0.92116 & 2.27684\end{array}$

$1+$ aminomethane TS

$\begin{array}{rrrr}\mathrm{Pt} & 0.35216 & -0.18642 & -0.01010 \\ \mathrm{~N} & 2.29390 & -0.19660 & 0.72211 \\ \mathrm{~N} & 0.02270 & -2.27802 & 0.05025 \\ \mathrm{Cl} & 0.61634 & 2.12927 & -0.21203 \\ \mathrm{~N} & -1.99393 & 0.46342 & 0.95400 \\ \mathrm{O} & -1.21845 & -0.34424 & -1.80911 \\ \mathrm{H} & -0.35643 & -2.60393 & 0.94130 \\ \mathrm{H} & 0.83584 & -2.85727 & -0.16539 \\ \mathrm{H} & -0.67860 & -2.46623 & -0.67167 \\ \mathrm{H} & 2.59365 & 0.78554 & 0.70822 \\ \mathrm{H} & 2.94999 & -0.72609 & 0.14467 \\ \mathrm{H} & 2.36748 & -0.52963 & 1.68545 \\ \mathrm{H} & -1.94739 & 0.26064 & -1.59136 \\ \mathrm{H} & -0.75258 & 0.07458 & -2.55493 \\ \mathrm{C} & -3.41581 & 0.20068 & 0.63621 \\ \mathrm{H} & -4.10571 & 0.63203 & 1.37191 \\ \mathrm{H} & -3.59045 & -0.87752 & 0.58641 \\ \mathrm{H} & -3.66586 & 0.63329 & -0.33826 \\ \mathrm{H} & -1.80400 & 1.46649 & 0.93178 \\ \mathrm{H} & -1.80814 & 0.17970 & 1.91513\end{array}$

$1+3$-methylthiophene TS
Pt -0.94463
0.30676
$\mathrm{N}-2.26368$
$\begin{array}{ll}1.59293 & 0.87618\end{array}$
$\mathrm{N} \quad 0.42602$
$\begin{array}{ll}1.80983 & -0.69488\end{array}$
$\mathrm{Cl}-2.40579$
$-1.41222$
0.52742
S 1.30243
$-1.34235$
0.29691
O $\quad-0.53329$
$-1.17714-2.04062$
C 2.56924
$-0.67540$
$-0.70633$
C 1.98236
$-0.59150$

$\begin{array}{rrrr}\mathrm{H} & 2.60275 & -0.92986 & -1.75795 \\ \mathrm{H} & 1.50649 & -0.73432 & 2.68241 \\ \mathrm{C} & 3.12431 & 0.08716 & 1.42544 \\ \mathrm{C} & 3.48372 & 0.03922 & 0.02379 \\ \mathrm{H} & 1.35515 & 1.37240 & -0.76688 \\ \mathrm{H} & 0.51231 & 2.58760 & -0.03891 \\ \mathrm{H} & 0.20168 & 2.20488 & -1.61043 \\ \mathrm{C} & 4.74165 & 0.65148 & -0.53083 \\ \mathrm{H} & 3.72339 & 0.59755 & 2.17273 \\ \mathrm{H} & -3.04334 & 0.99767 & 1.17928 \\ \mathrm{H} & -2.64510 & 2.32587 & 0.27522 \\ \mathrm{H} & -1.86531 & 2.02733 & 1.71095 \\ \mathrm{H} & -1.04955 & -1.91967 & -1.66818 \\ \mathrm{H} & -1.07181 & -0.86437 & -2.78865 \\ \mathrm{H} & 4.78374 & 0.57124 & -1.62039 \\ \mathrm{H} & 5.62455 & 0.14416 & -0.12392 \\ \mathrm{H} & 4.82800 & 1.70988 & -0.25824\end{array}$

$1+\mathrm{mG}$ product

$\begin{array}{llll}\text { Pt } & -1.65105 & -0.19526 & 0.07928\end{array}$

$\begin{array}{llll}\mathrm{Cl} & -2.20875 & 1.80056 & -1.04317\end{array}$

$\begin{array}{llll}\mathrm{N} & -3.64764 & -0.72586 & -0.25106\end{array}$

$\begin{array}{llll}\mathrm{N} & -1.19928 & -2.00095 & 1.07536\end{array}$

$\begin{array}{llll}\mathrm{N} & 0.28367 & 0.46561 & 0.31410\end{array}$

$\begin{array}{llll}\text { C } & 0.57068 & 1.74156 & 0.53548\end{array}$

$\begin{array}{llll}\text { C } & 1.50463 & -0.16389 & 0.07807\end{array}$

$\begin{array}{llll}\mathrm{N} & 1.90576 & 1.98431 & 0.45782\end{array}$

$\begin{array}{llll}\text { C } & 2.52008 & 0.78899 & 0.15845\end{array}$

$\begin{array}{llll}\text { C } & 2.57858 & 3.26992 & 0.64837\end{array}$

$\begin{array}{llll}\text { C } & 1.86666 & -1.52166 & -0.19069\end{array}$

$\begin{array}{llll}\mathrm{O} & 1.16622 & -2.53844 & -0.23363\end{array}$

$\begin{array}{llll}\mathrm{N} & 3.25704 & -1.63855 & -0.40367\end{array}$

$\begin{array}{llll}\text { C } & 4.17907 & -0.61607 & -0.32146\end{array}$

$\begin{array}{llll}\mathrm{N} & 3.84058 & 0.62502 & -0.02742\end{array}$

$\begin{array}{llll}\mathrm{N} & 5.48097 & -0.92834 & -0.51406\end{array}$

$\begin{array}{llll}\mathrm{H} & -1.01845 & -1.84204 & 2.06600\end{array}$

$\begin{array}{llll}\mathrm{H} & -0.33638 & -2.37557 & 0.63041\end{array}$

$\begin{array}{llll}\mathrm{H} & -1.92588 & -2.71149 & 1.01107\end{array}$

$\begin{array}{llll}\mathrm{H} & -0.16395 & 2.51229 & 0.70897\end{array}$

H $\quad 3.56278 \quad-2.59168 \quad-0.57419$

$\begin{array}{llll}\mathrm{H} & 3.12111 & 3.27369 & 1.59698\end{array}$

$\begin{array}{llll}\mathrm{H} & 3.28166 & 3.42953 & -0.17075\end{array}$

$\begin{array}{llll}\mathrm{H} & 1.82826 & 4.06147 & 0.64801\end{array}$

$\begin{array}{llll}\mathrm{H} & 6.13110 & -0.15485 & -0.54640\end{array}$

H $\quad 5.76312 \quad-1.78724 \quad-0.96323$

$\begin{array}{llll}\mathrm{H} & -4.07283 & 0.09025 & -0.70535\end{array}$ 


$\begin{array}{cccc}\mathrm{H} & -3.75501 & -1.51810 & -0.88794 \\ \mathrm{H} & -4.18096 & -0.91942 & 0.59882 \\ & & & \\ \mathbf{1}+\mathrm{H} \text { product } & & \\ \mathrm{Pt} & -0.86995 & -0.18715 & 0.01115 \\ \mathrm{~N} & -2.96146 & -0.25334 & -0.21376 \\ \mathrm{~N} & -0.65764 & -2.27900 & 0.38046 \\ \mathrm{Cl} & -1.10009 & 2.11228 & -0.33219 \\ \mathrm{~N} & 1.14550 & 0.02908 & 0.18368 \\ \mathrm{C} & 2.13341 & -0.61413 & -0.54965 \\ \mathrm{C} & 1.74926 & 0.91735 & 0.96519 \\ \mathrm{H} & 1.89783 & -1.35149 & -1.30165 \\ \mathrm{H} & 1.25693 & 1.60470 & 1.63457 \\ \mathrm{~N} & 3.08097 & 0.85177 & 0.76114 \\ \mathrm{C} & 3.35810 & -0.10874 & -0.19836 \\ \mathrm{H} & -1.17286 & -2.57676 & 1.21132 \\ \mathrm{H} & -0.96610 & -2.86420 & -0.39865 \\ \mathrm{H} & 0.32550 & -2.50125 & 0.55202 \\ \mathrm{C} & 4.73964 & -0.41713 & -0.67238 \\ \mathrm{H} & 3.76577 & 1.43386 & 1.22673 \\ \mathrm{H} & -3.24131 & 0.72601 & -0.34216 \\ \mathrm{H} & -3.27098 & -0.76650 & -1.04160 \\ \mathrm{H} & -3.47247 & -0.61349 & 0.59441 \\ \mathrm{H} & 4.70666 & -1.19456 & -1.43933 \\ \mathrm{H} & 5.21828 & 0.46562 & -1.11193 \\ \mathrm{H} & 5.37676 & -0.77645 & 0.14432 \\ & & & \end{array}$

$\begin{array}{llll}\mathrm{H} & -2.32352 & 1.15559 & 1.35748\end{array}$

$\mathbf{1}+\mathrm{C}^{-}$product

Pt $0.30926 \quad-0.17846 \quad-0.02662$

$\begin{array}{llll}\mathrm{N} & 2.45830 & 0.09253 & 0.30579\end{array}$

$\begin{array}{llll}\mathrm{N} & 0.25643 & -2.28703 & 0.12649\end{array}$

$\begin{array}{llll}\mathrm{Cl} & 0.31699 & 2.17622 & -0.15210\end{array}$

$\begin{array}{llll}\text { S } & -1.97571 & -0.40936 & -0.48146\end{array}$

$\begin{array}{llll}\mathrm{H} & -0.75071 & -2.49733 & 0.08827\end{array}$

$\begin{array}{llll}\mathrm{H} & 0.71914 & -2.76890 & -0.64530\end{array}$

$\begin{array}{llll}\mathrm{H} & 0.62795 & -2.64647 & 1.00591\end{array}$

$\begin{array}{llll}\mathrm{H} & 2.53921 & 1.11408 & 0.29692\end{array}$

$\begin{array}{llll}\mathrm{H} & 2.81391 & -0.24940 & 1.19773\end{array}$

$\begin{array}{llll}\mathrm{H} & 3.04326 & -0.27758 & -0.44288\end{array}$

$\begin{array}{llll}\text { C } & -2.81574 & 0.61645 & 0.79088\end{array}$

$\begin{array}{llll}\mathrm{H} & -3.88750 & 0.57625 & 0.57070\end{array}$

$\begin{array}{llll}\mathrm{H} & -2.64366 & 0.23666 & 1.80128\end{array}$

$\begin{array}{llll}\mathrm{H} & -2.46991 & 1.64933 & 0.72177\end{array}$

$1+$ aminomethane product

Pt $0.15206 \quad-0.18120 \quad-0.05043$

$\begin{array}{llll}\mathrm{N} & 2.18749 & -0.46443 & 0.38874\end{array}$

$\begin{array}{llll}\mathrm{N} & -0.26933 & -2.28136 & -0.08610\end{array}$

$\begin{array}{llll}\mathrm{Cl} & 0.61296 & 2.10518 & -0.03918\end{array}$

$\begin{array}{llll}\mathrm{N} & -1.82195 & 0.35187 & -0.48487\end{array}$

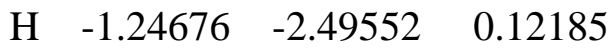

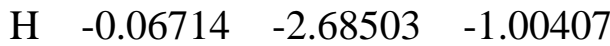

$\begin{array}{llll}\mathrm{H} & 0.28098 & -2.80508 & 0.59772\end{array}$

$\begin{array}{llll}\mathrm{H} & 2.59823 & 0.47606 & 0.37306\end{array}$

$\begin{array}{llll}\mathrm{H} & 2.36353 & -0.85092 & 1.31885\end{array}$

$\begin{array}{llll}\mathrm{H} & 2.69742 & -1.02799 & -0.29479\end{array}$

$\begin{array}{llll}\text { C } & -2.77325 & 0.37423 & 0.66792\end{array}$

$\begin{array}{llll}\mathrm{H} & -1.73040 & 1.30607 & -0.84924\end{array}$

$\begin{array}{llll}\mathrm{H} & -3.75460 & 0.72915 & 0.34019\end{array}$

$\begin{array}{llll}\mathrm{H} & -2.87092 & -0.63008 & 1.08453\end{array}$

$\begin{array}{llll}\mathrm{H} & -2.37443 & 1.04202 & 1.43173\end{array}$

$\begin{array}{llll}\mathrm{H} & -2.21107 & -0.20109 & -1.25183\end{array}$

$\begin{array}{llll}\mathrm{H} & 2.43828 & 1.58284 & 0.00002\end{array}$

$\begin{array}{llll}\mathrm{H} & 3.04189 & 0.28965 & 0.82844\end{array}$

$\begin{array}{llll}\mathrm{H} & 3.04190 & 0.28968 & -0.82845\end{array}$

$\begin{array}{llll}\text { C } & -2.53824 & 0.08778 & 1.41453\end{array}$

$\begin{array}{llll}\text { C } & -2.53825 & 0.08776 & -1.41453\end{array}$

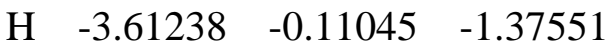

$\begin{array}{llll}\mathrm{H} & -2.32353 & 1.15557 & -1.35749\end{array}$

$\begin{array}{llll}\mathrm{H} & -2.11635 & -0.33751 & -2.32700\end{array}$

$\begin{array}{llll}\mathrm{H} & -3.61237 & -0.11043 & 1.37553\end{array}$

$\begin{array}{llll}\mathrm{H} & -2.11633 & -0.33749 & 2.32700\end{array}$

$1+3$-methylthiophene product

$\begin{array}{rrrc}\mathrm{Pt} & -0.89706 & -0.26456 & -0.02749 \\ \mathrm{~N} & -2.78551 & -0.98542 & 0.56632 \\ \mathrm{~N} & 0.03925 & -2.17339 & 0.08328 \\ \mathrm{Cl} & -1.93174 & 1.83519 & -0.08766 \\ \mathrm{~S} & 1.07326 & 0.84533 & -0.74253 \\ \mathrm{C} & 2.44124 & -0.26207 & -0.89883 \\ \mathrm{C} & 1.72488 & 1.49730 & 0.76990\end{array}$




$\begin{array}{crrc}\mathrm{H} & 2.54453 & -0.85612 & -1.79883 \\ \mathrm{H} & 1.15766 & 2.25110 & 1.29917 \\ \mathrm{C} & 2.93279 & 0.95803 & 1.03984 \\ \mathrm{C} & 3.37148 & -0.04317 & 0.07285 \\ \mathrm{H} & -0.15277 & -2.66254 & 0.95966 \\ \mathrm{H} & -0.23662 & -2.79074 & -0.68367 \\ \mathrm{H} & 1.05527 & -2.05070 & 0.02316 \\ \mathrm{C} & 4.71762 & -0.70342 & 0.14452 \\ \mathrm{H} & 3.53476 & 1.24962 & 1.89445 \\ \mathrm{H} & -3.37868 & -0.14839 & 0.61042 \\ \mathrm{H} & -3.20919 & -1.62613 & -0.10788 \\ \mathrm{H} & -2.80455 & -1.42568 & 1.48822 \\ \mathrm{H} & 4.84574 & -1.45395 & -0.63932 \\ \mathrm{H} & 5.51310 & 0.04333 & 0.03433 \\ \mathrm{H} & 4.86476 & -1.18586 & 1.11815\end{array}$

$2+$ mG TS (with $\mathrm{O}-\mathrm{H}$... O bond)

$\begin{array}{rrrr}\mathrm{Pt} & -1.89237 & -0.10114 & -0.05247 \\ \mathrm{~N} & -2.55227 & 1.66589 & -0.93466 \\ \mathrm{~N} & -3.00888 & -1.38132 & -1.23110 \\ \mathrm{O} & -1.28393 & -1.86995 & 0.78073 \\ \mathrm{O} & -1.67461 & 1.42398 & 1.81832 \\ \mathrm{~N} & 0.54337 & 0.76712 & 0.25352 \\ \mathrm{C} & 1.67468 & -0.03686 & 0.13759 \\ \mathrm{C} & 1.87048 & -1.43454 & 0.20982 \\ \mathrm{~N} & 3.19649 & -1.80874 & 0.03946 \\ \mathrm{C} & 4.25269 & -0.93517 & -0.17477 \\ \mathrm{~N} & 4.07868 & 0.37684 & -0.23561 \\ \mathrm{C} & 2.80989 & 0.76065 & -0.07773 \\ \mathrm{~N} & 2.35681 & 2.05839 & -0.09014 \\ \mathrm{C} & 1.00528 & 1.99862 & 0.11041 \\ \mathrm{C} & 3.18723 & 3.25452 & -0.27458 \\ \mathrm{O} & 1.03305 & -2.36185 & 0.40150 \\ \mathrm{~N} & 5.47814 & -1.45601 & -0.32310 \\ \mathrm{H} & -0.16377 & -2.07848 & 0.62115 \\ \mathrm{H} & -1.52511 & -1.97109 & 1.71377 \\ \mathrm{H} & 3.36163 & -2.81085 & 0.08941 \\ \mathrm{H} & 5.67975 & -2.44441 & -0.27473 \\ \mathrm{H} & 6.24968 & -0.81848 & -0.47212 \\ \mathrm{H} & 0.40612 & 2.89856 & 0.15321 \\ \mathrm{H} & -2.28867 & 1.31623 & 2.56662 \\ \mathrm{H} & -0.77324 & 1.33906 & 2.17571 \\ \mathrm{H} & -4.02171 & -1.26352 & -1.14221 \\ \mathrm{H} & -2.77568 & -2.31927 & -0.88309 \\ \mathrm{H} & -2.77133 & -1.35143 & -2.22624 \\ \mathrm{H} & -2.50234 & 2.39770 & -0.21837\end{array}$

$$
\begin{array}{rrrr}
\mathrm{H} & -3.51876 & 1.64892 & -1.27031 \\
\mathrm{H} & -1.96752 & 1.95982 & -1.72172 \\
\mathrm{H} & 3.96806 & 3.27734 & 0.48810 \\
\mathrm{H} & 3.64804 & 3.23388 & -1.26446 \\
\mathrm{H} & 2.55582 & 4.13881 & -0.17983
\end{array}
$$

$2+$ mG TS (with N-H...O bond)

$\begin{array}{llll}\mathrm{Pt} & -1.89548 & -0.07196 & -0.04618\end{array}$

$\begin{array}{llll}\mathrm{N} & -1.49692 & -1.81746 & 0.89428\end{array}$

$\begin{array}{llll}\mathrm{N} & -3.05435 & -1.03875 & -1.49339\end{array}$

$\begin{array}{llll}\mathrm{O} & -2.22440 & 1.87639 & -0.90718\end{array}$

$\begin{array}{llll}\mathrm{O} & -1.52314 & 0.70394 & 2.16234\end{array}$

$\begin{array}{llll}\mathrm{N} & 0.53145 & 0.72423 & 0.23680\end{array}$

$\begin{array}{llll}\text { C } & 0.98316 & 1.96884 & 0.16129\end{array}$

$\begin{array}{llll}\mathrm{N} & 2.33694 & 2.04038 & -0.00563\end{array}$

$\begin{array}{llll}\text { C } & 2.79720 & 0.74226 & -0.04487\end{array}$

$\begin{array}{llll}\text { C } & 1.66714 & -0.06737 & 0.10666\end{array}$

$\begin{array}{llll}\text { C } & 3.16720 & 3.24432 & -0.11541\end{array}$

$\begin{array}{llll}\mathrm{N} & 4.07409 & 0.37372 & -0.19802\end{array}$

$\begin{array}{llll}\text { C } & 4.24817 & -0.93949 & -0.20783\end{array}$

$\begin{array}{llll}\mathrm{N} & 5.48196 & -1.44574 & -0.35804\end{array}$

$\begin{array}{llll}\mathrm{N} & 3.19456 & -1.82901 & -0.07336\end{array}$

$\begin{array}{llll}\text { C } & 1.84664 & -1.48113 & 0.09172\end{array}$

$\begin{array}{llll}\mathrm{O} & 0.99920 & -2.38710 & 0.19680\end{array}$

$\begin{array}{llll}\mathrm{H} & -2.12629 & -2.59636 & 0.69776\end{array}$

$\begin{array}{llll}\mathrm{H} & -0.49553 & -2.09564 & 0.64097\end{array}$

$\begin{array}{llll}\mathrm{H} & -1.54659 & -1.62875 & 1.89956\end{array}$

$\begin{array}{llll}\mathrm{H} & 3.36672 & -2.83046 & -0.08698\end{array}$

H $\quad 5.68867 \quad-2.43366 \quad-0.34170$

$\begin{array}{llll}\mathrm{H} & 6.25350 & -0.79709 & -0.44118\end{array}$

$\begin{array}{llll}\mathrm{H} & 0.37054 & 2.85898 & 0.21790\end{array}$

$\begin{array}{llll}\mathrm{H} & -2.15231 & 1.28957 & 2.61951\end{array}$

$\begin{array}{llll}\mathrm{H} & -0.64359 & 1.12175 & 2.19739\end{array}$

$\begin{array}{llll}\mathrm{H} & -2.51145 & -1.74392 & -2.00126\end{array}$

$\begin{array}{llll}\mathrm{H} & -3.41547 & -0.39097 & -2.19869\end{array}$

$\begin{array}{llll}\mathrm{H} & -3.87154 & -1.50742 & -1.09109\end{array}$

$\begin{array}{llll}\mathrm{H} & -1.80902 & 2.07746 & -1.76499\end{array}$

$\begin{array}{llll}\mathrm{H} & -3.11970 & 2.26106 & -0.90353\end{array}$

$\begin{array}{llll}\mathrm{H} & 3.67834 & 3.25173 & -1.08042\end{array}$

$\begin{array}{llll}\mathrm{H} & 2.52691 & 4.12335 & -0.03030\end{array}$

$\begin{array}{llll}\mathrm{H} & 3.90962 & 3.25147 & 0.68524\end{array}$

$\begin{array}{llrc}\mathbf{2}+\mathrm{H} \mathrm{TS} & & \\ \mathrm{Pt} & 1.12460 & -0.07334 & 0.00539 \\ \mathrm{~N} & 2.63756 & 0.63784 & -1.24018 \\ \mathrm{~N} & 0.73814 & -1.74377 & -1.10478\end{array}$ 


$\begin{array}{rrrr}\mathrm{O} & 1.50979 & 1.67145 & 1.16174 \\ \mathrm{~N} & -1.44600 & 0.29787 & 0.02644 \\ \mathrm{O} & 0.12523 & -1.32211 & 1.68499 \\ \mathrm{C} & -2.48567 & -0.61854 & 0.15978 \\ \mathrm{C} & -2.02336 & 1.43784 & -0.33647 \\ \mathrm{H} & -2.31494 & -1.64931 & 0.43864 \\ \mathrm{H} & -1.52396 & 2.37086 & -0.55662 \\ \mathrm{~N} & -3.36498 & 1.28382 & -0.42804 \\ \mathrm{C} & -3.69331 & -0.02213 & -0.11668 \\ \mathrm{H} & -0.07350 & -1.57984 & -1.71028 \\ \mathrm{H} & 1.50175 & -2.09736 & -1.68828 \\ \mathrm{H} & 0.48238 & -2.49409 & -0.45218 \\ \mathrm{C} & -5.09378 & -0.54007 & -0.11528 \\ \mathrm{H} & -4.02444 & 2.00835 & -0.68944 \\ \mathrm{H} & 2.93596 & 1.55747 & -0.89748 \\ \mathrm{H} & 3.47171 & 0.04308 & -1.25170 \\ \mathrm{H} & 2.33733 & 0.77178 & -2.21049 \\ \mathrm{H} & -0.71557 & -0.92920 & 1.98497 \\ \mathrm{H} & 0.62303 & -1.60706 & 2.47273 \\ \mathrm{H} & -5.10241 & -1.60057 & 0.14690 \\ \mathrm{H} & -5.71699 & -0.00900 & 0.61339 \\ \mathrm{H} & -5.56246 & -0.43343 & -1.10031 \\ \mathrm{H} & 0.70486 & 2.17535 & 1.39041 \\ \mathrm{H} & 2.00219 & 1.52560 & 1.99309\end{array}$

\section{$2+\mathrm{M}$ TS}

$\begin{array}{rrrr}\mathrm{Pt} & 0.70272 & 0.00678 & 0.01677 \\ \mathrm{~N} & 2.13123 & 0.55864 & -1.41522 \\ \mathrm{~N} & 0.89843 & -2.00418 & -0.30917 \\ \mathrm{O} & 0.57510 & 2.10071 & 0.36396 \\ \mathrm{~S} & -2.28146 & -0.16874 & 0.19052 \\ \mathrm{O} & 0.02244 & -0.85367 & 2.12777 \\ \mathrm{H} & 0.31883 & -2.34864 & -1.08123 \\ \mathrm{H} & 1.85820 & -2.31750 & -0.48563 \\ \mathrm{H} & 0.58562 & -2.47170 & 0.55127 \\ \mathrm{H} & 2.19127 & 1.58279 & -1.42511 \\ \mathrm{H} & 3.07337 & 0.21081 & -1.21101 \\ \mathrm{H} & 1.89082 & 0.26587 & -2.36711 \\ \mathrm{H} & -0.91481 & -0.77763 & 2.38789 \\ \mathrm{H} & 0.55961 & -0.72084 & 2.93011 \\ \mathrm{C} & -2.96512 & 1.50152 & -0.11959 \\ \mathrm{C} & -2.98463 & -1.10515 & -1.21840 \\ \mathrm{H} & -4.05621 & 1.43666 & -0.14975 \\ \mathrm{H} & -2.69621 & 2.13968 & 0.72761 \\ \mathrm{H} & -2.60081 & 1.92028 & -1.06189 \\ \mathrm{H} & -4.07476 & -1.04273 & -1.16799\end{array}$

$$
\begin{array}{cccc}
\mathrm{H} & -2.62801 & -0.71458 & -2.17387 \\
\mathrm{H} & -2.69434 & -2.15192 & -1.10026 \\
\mathrm{H} & -0.29438 & 2.51285 & 0.19739 \\
\mathrm{H} & 0.88361 & 2.41177 & 1.23755
\end{array}
$$$$
2+\mathrm{C}^{-} \mathrm{TS}
$$

$\begin{array}{llll}\text { Pt } & -0.59444 & 0.03621 & 0.07908\end{array}$

$\begin{array}{llll}\mathrm{N} & -2.19289 & -1.05200 & -0.75953\end{array}$

$\begin{array}{llll}\mathrm{N} & -0.95042 & 1.85306 & -0.82635\end{array}$

$\begin{array}{llll}\mathrm{O} & 0.00885 & -1.81385 & 0.88339\end{array}$

$\begin{array}{llll}\mathrm{S} & 2.09736 & -0.50355 & -0.60037\end{array}$

$\begin{array}{llll}\mathrm{O} & 0.74517 & 1.47679 & 1.36629\end{array}$

$\begin{array}{llll}\mathrm{H} & -0.46915 & 1.88707 & -1.72954\end{array}$

$\begin{array}{llll}\mathrm{H} & -1.92553 & 2.12213 & -0.96918\end{array}$

$\begin{array}{llll}\mathrm{H} & -0.51113 & 2.55517 & -0.22151\end{array}$

$\begin{array}{llll}\mathrm{H} & -2.07371 & -2.02126 & -0.45068\end{array}$

$\begin{array}{llll}\mathrm{H} & -3.12139 & -0.74404 & -0.46442\end{array}$

$\begin{array}{llll}\mathrm{H} & -2.16627 & -1.05765 & -1.78152\end{array}$

$\begin{array}{llll}\mathrm{H} & 1.56494 & 1.03510 & 1.01649\end{array}$

$\begin{array}{llll}\mathrm{H} & 0.73586 & 1.37511 & 2.33618\end{array}$

$\begin{array}{llll}\text { C } & 3.85221 & 0.05066 & -0.40890\end{array}$

$\begin{array}{llll}\mathrm{H} & 3.96963 & 1.04562 & -0.84495\end{array}$

$\begin{array}{llll}\mathrm{H} & 4.17503 & 0.06638 & 0.63446\end{array}$

$\begin{array}{llll}\mathrm{H} & 4.48029 & -0.64902 & -0.96609\end{array}$

$\begin{array}{llll}\mathrm{H} & 0.00697 & -1.84341 & 1.85908\end{array}$

$\begin{array}{llll}\mathrm{H} & 1.00122 & -1.75384 & 0.57636\end{array}$

$2+\mathrm{mG}$ product (with $\mathrm{O}-\mathrm{H}$...O bond)

$\begin{array}{llll}\mathrm{Pt} & 1.72610 & -0.00405 & -0.00758\end{array}$

$\begin{array}{llll}\mathrm{O} & 1.27684 & -1.73125 & -1.04473\end{array}$

$\begin{array}{llll}\mathrm{N} & 3.64076 & -0.85818 & 0.09593\end{array}$

$\begin{array}{llll}\mathrm{N} & 2.25920 & 1.64165 & 1.16013\end{array}$

$\begin{array}{llll}\mathrm{N} & -0.21555 & 0.67160 & -0.17123\end{array}$

$\begin{array}{llll}\text { C } & -0.63497 & 1.92259 & -0.35499\end{array}$

$\begin{array}{llll}\mathrm{N} & -1.98587 & 2.02586 & -0.30424\end{array}$

$\begin{array}{llll}\text { C } & -2.48378 & 0.75783 & -0.07512\end{array}$

$\begin{array}{llll}\text { C } & -1.37488 & -0.09083 & -0.01057\end{array}$

$\begin{array}{llll}\text { C } & -2.79672 & 3.24102 & -0.47786\end{array}$

$\begin{array}{llll}\mathrm{N} & -3.77209 & 0.44371 & 0.04975\end{array}$

$\begin{array}{llll}\text { C } & -3.99425 & -0.84996 & 0.25209\end{array}$

$\begin{array}{llll}\mathrm{N} & -2.96373 & -1.77886 & 0.27692\end{array}$

$\begin{array}{llll}\text { C } & -1.61284 & -1.48856 & 0.10932\end{array}$

$\begin{array}{llll}\text { O } & -0.79370 & -2.43412 & 0.05563\end{array}$

$\begin{array}{llll}\mathrm{N} & -5.24402 & -1.29088 & 0.42683\end{array}$

$\mathrm{H} \quad 1.11116 \quad-1.59451 \quad-1.99158$

H $\quad 0.39240 \quad-2.12793 \quad-0.58230$ 


$\begin{array}{rrrr}\mathrm{H} & 4.37169 & -0.33138 & -0.39086 \\ \mathrm{H} & 3.56451 & -1.76765 & -0.37361 \\ \mathrm{H} & 3.97755 & -1.04954 & 1.04367 \\ \mathrm{H} & 2.79194 & 2.34331 & 0.63615 \\ \mathrm{H} & 2.83273 & 1.37603 & 1.96632 \\ \mathrm{H} & 1.43838 & 2.11365 & 1.54921 \\ \mathrm{H} & -3.17009 & -2.77144 & 0.36410 \\ \mathrm{H} & -5.48181 & -2.25893 & 0.59281 \\ \mathrm{H} & -5.99648 & -0.61404 & 0.39905 \\ \mathrm{H} & 0.00334 & 2.77376 & -0.54399 \\ \mathrm{H} & -3.44578 & 3.12203 & -1.34754 \\ \mathrm{H} & -3.40634 & 3.39732 & 0.41407 \\ \mathrm{H} & -2.13044 & 4.09122 & -0.62733\end{array}$

$2+\mathrm{mG}$ product (with $\mathrm{N}-\mathrm{H}$... O bond)

$\begin{array}{llll}\mathrm{Pt} & 1.72146 & -0.02339 & -0.02607\end{array}$

$\begin{array}{llll}\mathrm{N} & 1.28195 & -1.51961 & -1.34031\end{array}$

$\begin{array}{llll}\mathrm{N} & 3.70604 & -0.67770 & 0.22871\end{array}$

$\begin{array}{llll}\mathrm{O} & 2.24515 & 1.45571 & 1.44177\end{array}$

$\begin{array}{llll}\mathrm{N} & -0.22014 & 0.63284 & -0.20426\end{array}$

$\begin{array}{llll}\text { C } & -1.38700 & -0.10861 & -0.01695\end{array}$

$\begin{array}{llll}\text { C } & -2.48092 & 0.75298 & -0.08282\end{array}$

$\begin{array}{llll}\mathrm{N} & -1.96581 & 2.01268 & -0.33208\end{array}$

$\begin{array}{llll}\text { C } & -0.61801 & 1.89111 & -0.39684\end{array}$

$\begin{array}{llll}\text { C } & -2.76197 & 3.23567 & -0.51219\end{array}$

$\begin{array}{llll}\text { C } & -1.62766 & -1.50943 & 0.13952\end{array}$

$\begin{array}{llll}\mathrm{N} & -2.99325 & -1.77144 & 0.31960\end{array}$

$\begin{array}{llll}\text { C } & -4.00916 & -0.82936 & 0.27948\end{array}$

$\begin{array}{cccc}\mathrm{N} & -3.77443 & 0.45906 & 0.05747\end{array}$

$\begin{array}{llll}\mathrm{N} & -5.26627 & -1.25046 & 0.46248\end{array}$

$\begin{array}{llll}\mathrm{O} & -0.81150 & -2.44110 & 0.10199\end{array}$

$\begin{array}{llll}\mathrm{H} & 0.95507 & -1.15038 & -2.23554\end{array}$

$\mathrm{H} \quad 0.50106 \quad-2.05924 \quad-0.87931$

$\begin{array}{llll}\mathrm{H} & 2.05371 & -2.15638 & -1.54177\end{array}$

$\mathrm{H} \quad 4.16967 \quad-0.08115 \quad 0.92145$

$\begin{array}{llll}\mathrm{H} & 4.27304 & -0.62954 & -0.62322\end{array}$

$\begin{array}{llll}\mathrm{H} & 3.77129 & -1.63562 & 0.58674\end{array}$

$\mathrm{H} \quad 2.33456 \quad 2.38438 \quad 1.15789$

$\begin{array}{llll}\mathrm{H} & 1.70599 & 1.44773 & 2.25525\end{array}$

$\begin{array}{llll}\mathrm{H} & -3.21450 & -2.75737 & 0.43521\end{array}$

$\mathrm{H} \quad-5.51572 \quad-2.21225 \quad 0.64477$

$\begin{array}{llll}\mathrm{H} & -6.00891 & -0.56382 & 0.42625\end{array}$

$\begin{array}{llll}\mathrm{H} & 0.03334 & 2.72904 & -0.59760\end{array}$

$\begin{array}{llll}\mathrm{H} & -3.42373 & 3.11337 & -1.37185\end{array}$

$\begin{array}{llll}\mathrm{H} & -3.35940 & 3.41204 & 0.38423\end{array}$

$\begin{array}{llll}\mathrm{H} & -2.08702 & 4.07528 & -0.68156\end{array}$

$$
\begin{array}{lrrr}
\text { 2 + H product } & & \\
\mathrm{Pt} & -0.92624 & -0.01968 & -0.01289 \\
\mathrm{~N} & -3.02520 & 0.08168 & -0.27065 \\
\mathrm{~N} & -0.88120 & -2.07618 & -0.02692 \\
\mathrm{O} & -1.02458 & 2.10773 & -0.02625 \\
\mathrm{~N} & 1.10059 & -0.02082 & 0.21417 \\
\mathrm{C} & 2.03441 & -0.01042 & -0.82005 \\
\mathrm{C} & 1.78036 & 0.00065 & 1.36601 \\
\mathrm{H} & 1.74031 & -0.02495 & -1.85862 \\
\mathrm{H} & 1.36003 & -0.00385 & 2.36080 \\
\mathrm{~N} & 3.09601 & 0.02682 & 1.09019 \\
\mathrm{C} & 3.29620 & 0.02053 & -0.28190 \\
\mathrm{H} & -1.39555 & -2.49084 & 0.75751 \\
\mathrm{H} & -1.26595 & -2.47523 & -0.88973 \\
\mathrm{H} & 0.08547 & -2.41048 & 0.04382 \\
\mathrm{C} & 4.64868 & 0.04424 & -0.91312 \\
\mathrm{H} & 3.83259 & 0.04583 & 1.78872 \\
\mathrm{H} & -3.30009 & 1.06845 & -0.32447 \\
\mathrm{H} & -3.36566 & -0.35539 & -1.13256 \\
\mathrm{H} & -3.56114 & -0.32311 & 0.50321 \\
\mathrm{H} & 4.55532 & 0.03016 & -2.00124 \\
\mathrm{H} & 5.20299 & 0.94716 & -0.63304 \\
\mathrm{H} & 5.24334 & -0.82623 & -0.61368 \\
\mathrm{H} & -0.75927 & 2.54553 & 0.80617 \\
\mathrm{H} & -0.51848 & 2.53580 & -0.74466
\end{array}
$$

$2+$ M product

$\begin{array}{llll}\text { Pt } & -0.50023 & -0.01662 & 0.00000\end{array}$

$\begin{array}{llll}\mathrm{N} & -2.58456 & 0.44850 & -0.00000\end{array}$

$\begin{array}{llll}\mathrm{N} & -0.87437 & -2.04651 & -0.00001\end{array}$

$\begin{array}{llll}\mathrm{O} & -0.27580 & 2.10433 & 0.00001\end{array}$

$\begin{array}{llll}\mathrm{S} & 1.81760 & -0.57930 & 0.00000\end{array}$

$\begin{array}{llll}\mathrm{H} & -0.01260 & -2.60459 & 0.00001\end{array}$

$\begin{array}{llll}\mathrm{H} & -1.40739 & -2.33969 & 0.82658\end{array}$

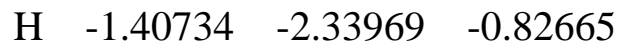

$\begin{array}{llll}\mathrm{H} & -2.68829 & 1.46871 & -0.00001\end{array}$

$\begin{array}{llll}\mathrm{H} & -3.08812 & 0.10809 & -0.82508\end{array}$

$\begin{array}{llll}\mathrm{H} & -3.08812 & 0.10811 & 0.82509\end{array}$

$\begin{array}{llll}\text { C } & 2.60472 & 0.25485 & -1.42882\end{array}$

$\begin{array}{llll}\text { C } & 2.60472 & 0.25486 & 1.42882\end{array}$

H $\quad 3.66044 \quad-0.03150 \quad 1.42570$

$\begin{array}{llll}\mathrm{H} & 2.51026 & 1.34017 & 1.35715\end{array}$

H $\quad 2.12995 \quad-0.12130 \quad 2.33735$

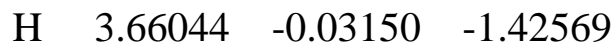

H $\quad 2.12995 \quad-0.12132 \quad-2.33735$ 


\begin{tabular}{crrr}
$\mathrm{H}$ & 2.51026 & 1.34016 & -1.35716 \\
$\mathrm{H}$ & 0.09451 & 2.54149 & 0.79055 \\
$\mathrm{H}$ & 0.09452 & 2.54150 & -0.79051 \\
\multicolumn{4}{c}{} \\
$\mathbf{2}+\mathrm{C}^{-}$product & & \\
$\mathrm{Pt}$ & 0.33934 & 0.01862 & -0.04387 \\
$\mathrm{~N}$ & 2.48909 & -0.03533 & 0.46920 \\
$\mathrm{~N}$ & 0.20498 & 2.06811 & -0.16692 \\
$\mathrm{O}$ & 0.29134 & -2.11605 & 0.01730 \\
$\mathrm{~S}$ & -1.92844 & -0.13234 & -0.58153 \\
$\mathrm{H}$ & -0.71718 & 2.28207 & -0.56526 \\
$\mathrm{H}$ & 0.26677 & 2.53469 & 0.74149 \\
$\mathrm{H}$ & 0.90740 & 2.48034 & -0.78538 \\
$\mathrm{H}$ & 2.69993 & -0.93935 & 0.89731 \\
$\mathrm{H}$ & 3.10391 & 0.06239 & -0.34130 \\
$\mathrm{H}$ & 2.77116 & 0.67351 & 1.14815 \\
$\mathrm{C}$ & -2.79559 & 0.09868 & 1.03305 \\
$\mathrm{H}$ & -3.85125 & -0.10513 & 0.83089 \\
$\mathrm{H}$ & -2.44051 & -0.59879 & 1.79378 \\
$\mathrm{H}$ & -2.70468 & 1.12410 & 1.39824 \\
$\mathrm{H}$ & -0.68443 & -2.26835 & -0.01326 \\
$\mathrm{H}$ & 0.61996 & -2.47323 & -0.83093
\end{tabular}

$\mathbf{1}+\mathrm{NH}_{3} \mathrm{TS}$

$\begin{array}{rrrc}\mathrm{Pt} & 0.11260 & -0.20212 & 0.01540 \\ \mathrm{~N} & -0.67045 & -2.12607 & -0.25329 \\ \mathrm{~N} & 2.19194 & -0.71309 & -0.04859 \\ \mathrm{Cl} & -2.13626 & 0.49407 & 0.18594 \\ \mathrm{~N} & 0.38070 & 1.94271 & -1.48653 \\ \mathrm{O} & 0.90777 & 1.61197 & 1.40414 \\ \mathrm{H} & 2.56917 & -0.75752 & -1.00592 \\ \mathrm{H} & 2.43880 & -1.59402 & 0.42376 \\ \mathrm{H} & 2.68790 & 0.04419 & 0.44628 \\ \mathrm{H} & -1.69742 & -2.00966 & -0.18868 \\ \mathrm{H} & -0.38441 & -2.79586 & 0.47514 \\ \mathrm{H} & -0.45578 & -2.53089 & -1.17618 \\ \mathrm{H} & 0.43962 & 2.39396 & 1.03529 \\ \mathrm{H} & 0.43605 & 1.42557 & 2.24847 \\ \mathrm{H} & 1.00102 & 2.76465 & -1.44922 \\ \mathrm{H} & -0.59129 & 2.28185 & -1.46989 \\ \mathrm{H} & 0.51250 & 1.52357 & -2.41548\end{array}$

$1+\mathrm{H}_{2} \mathrm{~S}$ TS

$\begin{array}{llll}\text { Pt } & 0.29199 & -0.19626 & -0.04996\end{array}$

$\begin{array}{llll}\mathrm{N} & 2.23234 & -0.31919 & 0.77417\end{array}$

$\begin{array}{llll}\mathrm{N} & -0.02147 & -2.31294 & -0.23146\end{array}$

$\begin{array}{crrr}\mathrm{Cl} & 0.53796 & 2.14788 & 0.09784 \\ \mathrm{~S} & -2.41821 & -0.00997 & 0.97148 \\ \mathrm{O} & -1.15450 & 0.38433 & -1.97391 \\ \mathrm{H} & -0.39746 & -2.70515 & 0.64444 \\ \mathrm{H} & 0.81626 & -2.85774 & -0.48036 \\ \mathrm{H} & -0.72203 & -2.49452 & -0.96543 \\ \mathrm{H} & 2.53390 & 0.66199 & 0.90344 \\ \mathrm{H} & 2.91595 & -0.77784 & 0.15512 \\ \mathrm{H} & 2.25596 & -0.77990 & 1.69520 \\ \mathrm{H} & -1.02547 & 1.35346 & -1.81825 \\ \mathrm{H} & -0.61217 & 0.21074 & -2.77739 \\ \mathrm{H} & -3.11012 & 0.45615 & -0.10549 \\ \mathrm{H} & -2.12395 & 1.23688 & 1.43117\end{array}$

$2+\mathrm{NH}_{3} \mathrm{TS}$

$\begin{array}{llll}\text { Pt } & -0.15852 & -0.04314 & -0.04151\end{array}$

$\begin{array}{llll}\mathrm{N} & -2.20444 & -0.35181 & 0.35410\end{array}$

$\begin{array}{llll}\mathrm{N} & 0.37856 & -2.02208 & 0.22371\end{array}$

$\begin{array}{llll}\mathrm{O} & -0.79463 & 1.99189 & -0.36670\end{array}$

$\begin{array}{llll}\mathrm{N} & 1.91615 & 0.79890 & 1.47489\end{array}$

$\begin{array}{llll}\mathrm{O} & 1.82382 & -0.16232 & -1.36564\end{array}$

$\mathrm{H} \quad 0.51706 \quad-2.27678 \quad 1.21600$

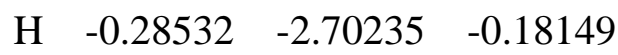

$\begin{array}{llll}\mathrm{H} & 1.28210 & -2.15042 & -0.26677\end{array}$

$\begin{array}{llll}\mathrm{H} & -2.68243 & 0.56292 & 0.28418\end{array}$

$\begin{array}{llll}\mathrm{H} & -2.66013 & -0.98267 & -0.32523\end{array}$

$\begin{array}{llll}\mathrm{H} & -2.38814 & -0.71817 & 1.30233\end{array}$

$\begin{array}{llll}\mathrm{H} & 2.55896 & 0.46444 & -1.17836\end{array}$

$\begin{array}{llll}\mathrm{H} & 1.69051 & -0.13805 & -2.34203\end{array}$

$\begin{array}{llll}\mathrm{H} & 2.70533 & 0.14371 & 1.57954\end{array}$

$\begin{array}{llll}\mathrm{H} & 2.34833 & 1.72365 & 1.32677\end{array}$

$\begin{array}{llll}\mathrm{H} & 1.48246 & 0.85035 & 2.40795\end{array}$

$\begin{array}{llll}\mathrm{H} & -0.35553 & 2.66697 & 0.20306\end{array}$

$\begin{array}{llll}\mathrm{H} & -0.71380 & 2.30942 & -1.29823\end{array}$

$2+\mathrm{H}_{2} \mathrm{~S}$ TS

$\begin{array}{llll}\text { Pt } & -0.32662 & 0.02648 & 0.02562\end{array}$

$\begin{array}{llll}\mathrm{N} & -2.23234 & -0.55754 & -0.69418\end{array}$

$\begin{array}{llll}\mathrm{N} & -0.37932 & 1.92664 & -0.79027\end{array}$

$\begin{array}{llll}\mathrm{O} & -0.32345 & -1.94583 & 0.89748\end{array}$

$\begin{array}{llll}\text { S } & 2.44150 & -0.59837 & -0.71212\end{array}$

$\begin{array}{llll}\mathrm{O} & 0.98509 & 1.32848 & 1.68020\end{array}$

$\begin{array}{llll}\mathrm{H} & 0.00824 & 1.96349 & -1.74766\end{array}$

$\begin{array}{llll}\mathrm{H} & -1.31741 & 2.35867 & -0.82119\end{array}$

$\begin{array}{llll}\mathrm{H} & 0.21328 & 2.52297 & -0.18370\end{array}$

$\begin{array}{llll}\mathrm{H} & -2.39047 & -1.53751 & -0.40322\end{array}$ 


$\begin{array}{rrrc}\mathrm{H} & -3.00837 & 0.00391 & -0.30691 \\ \mathrm{H} & -2.30090 & -0.53106 & -1.72440 \\ \mathrm{H} & 1.89550 & 1.10028 & 1.97808 \\ \mathrm{H} & 0.48958 & 1.52667 & 2.50852 \\ \mathrm{H} & 3.35613 & 0.40704 & -0.59072 \\ \mathrm{H} & 2.36278 & -0.48962 & -2.06728 \\ \mathrm{H} & 0.45064 & -2.50338 & 0.63881 \\ \mathrm{H} & -0.35834 & -1.95757 & 1.88478\end{array}$

